\title{
Application of Interferometry to Analysis of Polymer-Polymer and Polymer-Solvent Interactions
}

\author{
Veronica Makarova and Valery Kulichikhin \\ A.V. Topchiev Institute of Petrochemical Synthesis, Russian Academy of Sciences, \\ Russia
}

\section{Introduction}

For prediction of polymer systems behavior on processing and exploiting stages knowledge of phase diagrams is very useful and in some cases - necessary. They characterize equilibrium phase states and for particular systems - phase structure. Among other methods to estimate compatibility of two substances including polymeric one, and consequently to obtain reasonable information about phase state and structure of the final heterophase system, the optical interferometry method in a version of optical wedge (Borovskii et al, 1973; Malkin et al., 1983; Malkin\&Chalykh, 1979; Avdeev, 1990; Chalykh \& Gerasimov, 2004; Chalykh et al., 1998; Makarova, 2007) engages specific space. Each of components forms the intrinsic interference pattern with a pitch reciprocally proportional to its refraction index. Two components form two interference patterns separated by interface. As a result of interdiffusion, interference fringes close to the interface are bending. This process indicates on formation of solutions of various concentrations with their own refraction indices. Evolution of fringes shape in time gives the information about intensity of interpenetration of components allowing us to calculate the interdiffusion coefficients. Plotting concentration profiles at different temperatures and accepting that concentrations at the interface are equilibrium ones, it is possible to construct phase diagrams of binary systems.

In addition, kinetics of interference patterns evolution reflects constants of mass-transfer, i.e. kinetic stability of systems. This means that in one experiment we can obtain information on interaction kinetics and, finally, on composition of equilibrium phases. Such combination of kinetic and thermodynamic information is exclusively important for correct organization of blending stage and processing of polymer solutions, blends and alloys.

Simultaneously with high informativity, this method has some additional advantages compared with other methods for investigation of interdiffusion processes: simplicity, compactness of equipment, high enough preciseness of measurements, small amount of testing material. The main condition of this method consists in requirement that at least one of interacting components has to be transparent and difference of refraction indices should be not less than $10^{-3}$.

Method of optical interferometry in application to interaction of two polymeric components is based on multipath interference from two surfaces of the film (or films) under 
investigation (Fig.1). Films of testing polymers are placing in side-by-side (edge-to-edge) manner between two glass plates with a small wedge angle $\left(\sim 2^{\circ}\right)$ (Fig.2). Preliminary, semitransparent metallic layer $(\mathrm{Ag}, \mathrm{Au}, \mathrm{Ni}-\mathrm{Cr}$ alloy) is applied on inner surfaces of glass plates for reinforcing their reflection capability. At passing bunch of monochromatic beams through films of alternating thickness, the interference pattern appears as a result of pathlength difference.

In the beginning of experiment two systems of interference fringes (bands) with different pitches corresponding to refraction indices of each component are separated by interface. As a result of mutual dissolution, concentration gradients appear which lead to refraction index gradients and, consequently, to fringes bending. Evolution of fringes shape in time gives information about redistribution of components concentration in the diffusion zone as well as about the direction and the rate of the diffusion stream.

The optical interferometer used in this research was designed on a base of UV microscope (Avdeev, 1990; Makarova, 2007). The interferometer consists of light source, optical system, diffusion cell, oven and microscope equipped with video-camera. The diffusion cell consists of frame with optical channel, heating chamber, and the cell as it. Surface of glass plates with a thickness of 3-5 mm and plain dimensions $20 \times 25 \mathrm{~mm}$ is treated with the accuracy rating 14 . The necessary wedge angle and gap thickness was done by means of metallic (foil) ribbons with a thickness from 80 to $110 \mu$.

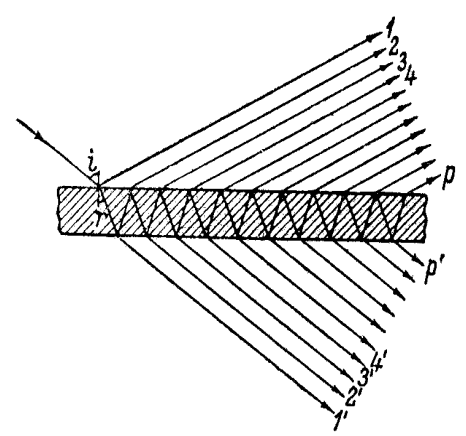

Fig. 1. Interference at multipass light refelection.

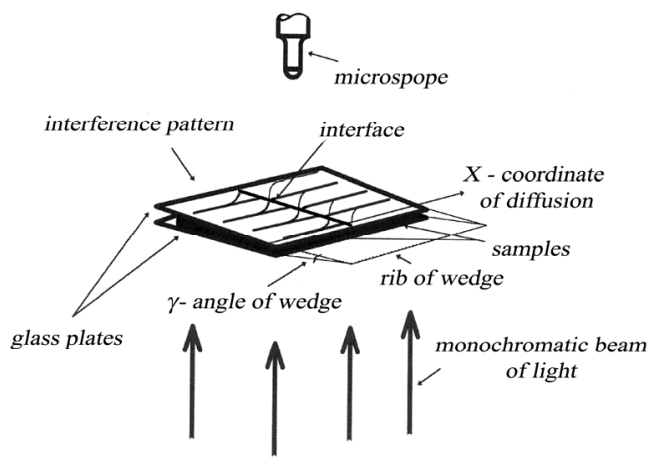

Fig. 2. Schematic image of the experimental procedure. 


\section{Results and discussion}

Binary polymer systems are thermodynamically reversible and submit to phase rule as lowmolecular weight formulations. The following kinds of the phase equilibrium are typical for these systems (the rank with increase of phase ordering) (Chalykh\&Gerasimov, 2004; Chalykh et al., 1998; Papkov, 1981; Tager, 1978; Lipatov, 1984; Klenin, 1995):

- $\quad$ amorphous (liquid-liquid) equilibrium realizing in the domain of bounded solubility of components where the system decomposes on two phases, and each of them is the saturated solution of one component into another;

- liquid-crystalline (mesomorphic) equilibrium characterizing with transition of isotropic solution to liquid crystal phase via biphasic zone where disordered and ordered phases are in equilibrium;

- crystalline equilibrium in that the saturated solution of one component into another coexists with crystalline or crystal solvate phase of this component.

In many cases the different kinds of the phase equilibrium can superimpose leading to complex phase equilibrium. Let consider in short each individual kind of the phase equilibrium and typical cases of their superposition.

\section{Amorphous equilibrium in polymer systems}

At mixing of two partially compatible liquids in the definite temperature-concentration domain a system decomposes on two liquid coexisting phases - saturated solutions of one component to another. Change of temperature results in change of components solubility and causes variation of coexisting phase compositions. At definite temperature, known as critical, boundaries between phases disappear and a system passes from heterophase to homophase. Return to previous conditions causes phase decomposition again. Such transitions can be performing unrestricted times without any shift of critical temperature.

Phase equilibria in binary systems are expressed by phase diagrams. Usually they are plotted in coordinates "temperature-composition" at constant pressure. Schematic images of basic kinds of phase diagrams of binary polymer systems characterizing with amorphous equilibrium are presented in Fig. 3. The equilibrium values of coexisting phases compositions form unique curve - binodal. Convex upwards binodal (Fig. 3a) corresponds to the case of increasing compatibility with increase of temperature. Its cupola reflects the limiting temperature higher that a system becomes single-phase at any concentration of components. This temperature is known as the Upper Critical Solubility Temperature (UCST). The phase composition corresponding to UCST is also named as critical.

If compatibility of components increases with decrease of temperature, the binodal has a shape of convex downwards curve (Fig. 3b) and critical temperature is named as Low Critical Solubility Temperature (LCST). Some systems can have both critical temperatures and their amorphous phase equilibriums are expressed by circle-like or closed (UCST>LCST) diagram (Fig. 3c), non-closed diagram (UCST<LCST) (Fig. 3d) or the diagram of sand-glass shape (UCMT LCMT) (Fig. 3f). 

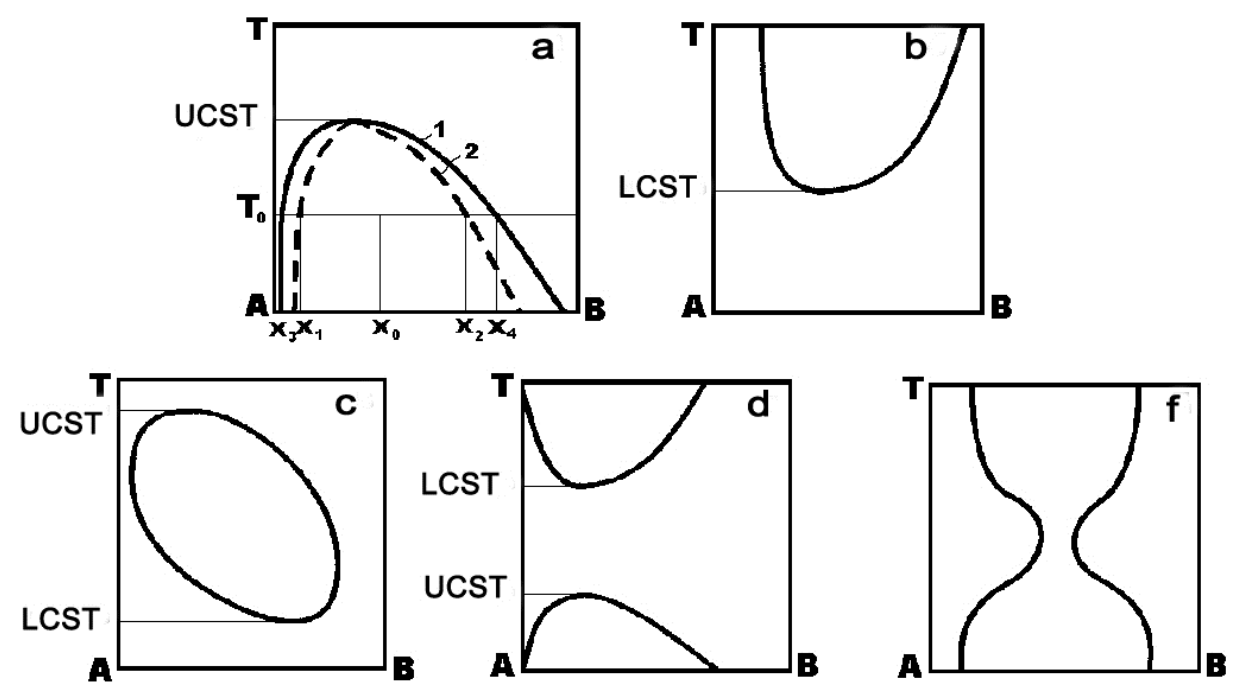

Fig. 3. Basic types of the phase diagrams of polymer system with amorphous equilibrium according to (Chalykh et al., 1998). See explanations in the text.

Under binodal a system decomposes on two phases. Analysis of kinetics of new phases formation indicates on unusual behavior of microheterophase system in vicinity of binodal and necessity to draw additional line - spinodal (Fig. 3a, curve 2). This line separates unstable (under binodal) and meta-stable (between binodal and spinodal) compositions. The critical point is the same as for binodal, as for spinodal. In meta-stable domain formation of novel phases proceeds very quickly at small fluctuations of composition.

In some cases also useful to show on phase diagrams boundaries of relaxation states of binary system (glass and brittleness points, flow temperature, limits of thermal stability of components, etc) (Papkov, 1971; Chalykh et al, 1990). Such diagrams are named "generalized phase diagrams" or "diagrams of phase and relaxation states".

Equilibrium curves shown in Fig. 3 are idealized. Actually, the total binodal curve cannot be registered ever because of temperature limitations. For example, in whole temperature diapason either the single-phase domain (complete compatibility of components) can exist or its coexistence with biphasic domain but without achievement of critical temperatures (partial compatibility of components). The most typical are phase diagrams with UCST because for majority systems increase of temperature leads to increase of kinetic mobility of molecules and macromolecules improving their mutual solubility. Diagrams with LCST meet for aqueous solutions of various polysaccharides

In the case of amorphous equilibrium at T>UCST (or T<LCST) the continuous change of composition at transition from one component to another is realized in diffusion zone. At temperatures of phase transitions in diffusion zone the border between two solutions appears. We will consider further some examples of amorphous phase equilibrium registered by interference method. As a rule, optical measurements were added by 
rheological data that allows us to obtain more informative picture of mass-transfer in vicinity of interfaces.

Amorphous phase equilibrium will be considered on examples of polycarbonate (PC) polymethylmethacrylate (PMMA) pair. PC and PMMA are transparent polymers (the coefficient of light transmission is $\sim 90 \%$ ) with similar exploiting properties. That is why fields of their application in many cases coincides (light-technical products, engineering plastics, materials for medicine, etc.). However, mechanical characteristics of PC, partially impact strength, are much better compared with PMMA. Keeping in mind that PC is more expensive than PMMA, development of blending materials based on these polymers can be interesting and important, but blends should be transparent and this means that the main task is to find temperature-composition regions of their compatibility.

Literature data concerning compatibility of these components are rather contradictive (Chiou et al, 1987; Kim\&Paul, 1992; Butzbach\&Wendorff, 1991; Landry \& Henrichs, 1989; Debier, 1994; Kyu, 1993; Nishimoto, 1991; Rabeony, 1992; Woo\&So, 1996): from conclusion about their immiscibility to partial or even total compatibility. Basic methods used in cited papers were light transmission, light scattering, DSC, and specimens for investigation were prepared from mutual for both polymers solutions. The nature of solvent, thermal prehistory and long relaxation times could influence significantly on formed heterophase structure (Woo\&So, 1996). Such uncertainty was the driving force for this investigation using interferometric method allowing us to observe direct penetration of melted components (Avdeev et al., 2001; Eastmond\&Kotomin, 1994).

Samples of PC $\left(\mathrm{Mw}=2.5 \cdot 10^{4} ; \mathrm{T}_{\mathrm{g}}=150^{\circ} \mathrm{C}, \mathrm{T}_{\mathrm{m}}=267^{\circ} \mathrm{C}\right)$ and series of PMMA $\left(\mathrm{M}_{\mathrm{w}}=1.5 \cdot 10^{4} ; 7.4 \cdot 10^{4}\right.$; $30.10^{4} ; \mathrm{T}_{\mathrm{g}}=114{ }^{\circ} \mathrm{C} ; \mathrm{T}_{\text {flow }}=180^{\circ} \mathrm{C}$ ) were used as objects under investigation. Experiments were carried out in temperature diapason from $150^{\circ} \mathrm{C}$ (glass point of PC) to $280^{\circ} \mathrm{C}$ (decomposition point of PMMA). Films with a thickness of 100-120 $\mu$ were prepared by hot-pressing at $150{ }^{\circ} \mathrm{C}$ (PMMA) and $240{ }^{\circ} \mathrm{C}$ (PMMA) with subsequent cooling with a rate of $5-10 \circ \mathrm{C} / \mathrm{min}$.

Viscosities of the neat and blended samples PC-PMMA $\left(\mathrm{M}_{\mathrm{W}}=1.5 \cdot 10^{4}\right)$ were measured by method of squeezing flow allowing us to work with micro-amount of materials. Interval of shear rates and stresses realized in this method at $240^{\circ} \mathrm{C}$ corresponds to Newtonian flow. Blends for rheological measurements were obtained via mutual solvent - chloroform that was removed in vacuum at $60^{\circ} \mathrm{C}$ during $8 \mathrm{~h}$.

Interferograms obtained present two interference patterns with a different pitches corresponding to the refraction indices of each component. In vicinity of interface "bands of equal thickness" bend that indicates on formation of solutions of various compositions PC in PMMA, on the one hand (Fig. 4, left) and PMMA in PC, on the other hand (right). Based on connection of the refraction index with composition, the profiles of concentration distribution in diffusion zone were plotted for different temperatures and observation times. Components concentrations reached on both sides of interface were accepted for solubility one into another.

Various curvature of bands near the interface for PMMA of different molecular weight reflects strong dependence of compatibility on $\mathrm{M}_{\mathrm{w}}$ PMMA. For interaction of PC with PMMA $\left(\mathrm{M}_{\mathrm{w}}=30 \cdot 10^{4}\right)$ the bands bending is practically absent and this means that the mutual dissolution of components is not higher than $0.5 \%$. In the case of PMMA with $\mathrm{M}_{\mathrm{w}}=7.4 \cdot 10^{4}$ the curvature is more significant but calculation shows that maximum solubility does not exceed 


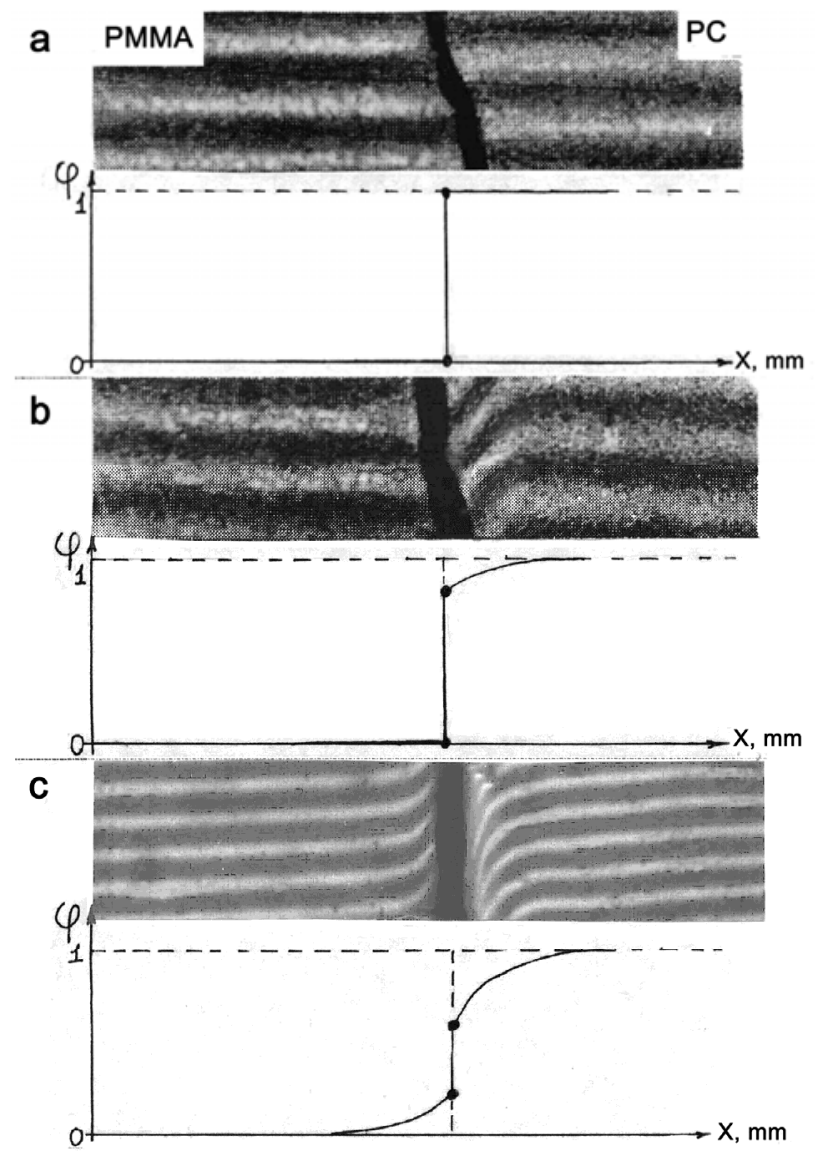

Fig. 4. Interferograms and concentration profiles at interaction of PC with PMMA $\left(\mathrm{M}_{\mathrm{W}}=30 \cdot 10^{4}(\mathrm{a}) ; 7 \cdot 4 \cdot 10^{4}(\mathrm{~b}) ; 1.5 \cdot 10^{4}(\mathrm{c})\right)$. Temperature $240^{\circ} \mathrm{C}$.

$3 \%$. The highest compatibility was observed for the system PC-PMMA with $\mathrm{M}_{\mathrm{w}}=1 \cdot 5 \cdot 10^{4}$. At permissible temperature of $280^{\circ} \mathrm{C}$ maximum solubility for PMMA in PC reaches $40 \%$ and for PC in PMMA - 20\%. This result corresponds to well-known, but subjective opinion about increase of mutual solubility for polymers, if one component has $\mathrm{M}_{\mathrm{W}}<10^{4}$ [3].

Using data on solubility of components at different temperatures the phase diagrams were plotted (Fig. 5). The temperature diapason was limited by glass point of PC and decomposition point of PMMA. One can see that there exists domains of mutual solubility of components and with growth of temperature the compatibility increases. Consequently, these diagrams belong to amorphous equilibrium with UCMT though the critical point was not achieved experimentally for any system because of decomposition of PMMA. Since the solubility of PMMA in PC is higher than vice versa, the binodal is asymmetric and shifted in direction of higher content of PMMA. 


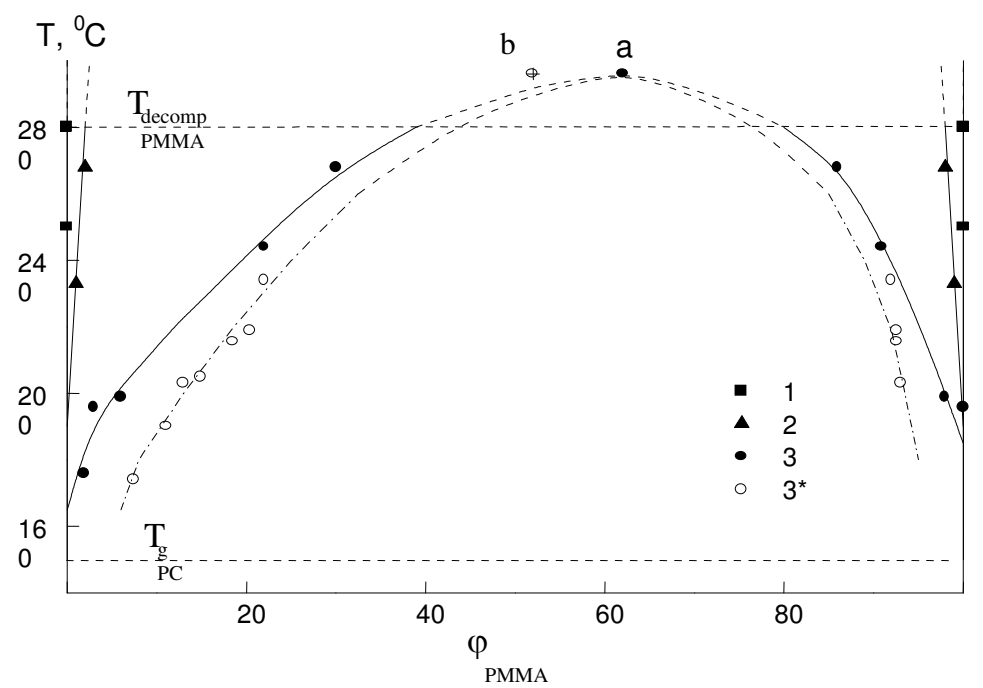

Fig. 5. Phase diagrams of PC-PMMA $\left(\mathrm{M}_{\mathrm{w}} \cdot 10^{-4}=30\right.$ (1), 7.4 (2) and 1.5 (3 - binodal, $3^{*}$ spinodal) systems. $a$ and $b$-calculated values of UCST with accepting of concentration dependence of $\chi$, and ignoring it, respectively.

For the system with lowest $\mathrm{M}_{\mathrm{W}}$ of PMMA the spinodal was determined as well. For this aim the blend was heated to $270^{\circ} \mathrm{C}$ for significant compatibility of components (Fig. 6a, b), and then it was cooled step-by-step keeping at each temperature several hours (Fig. 6c, d).

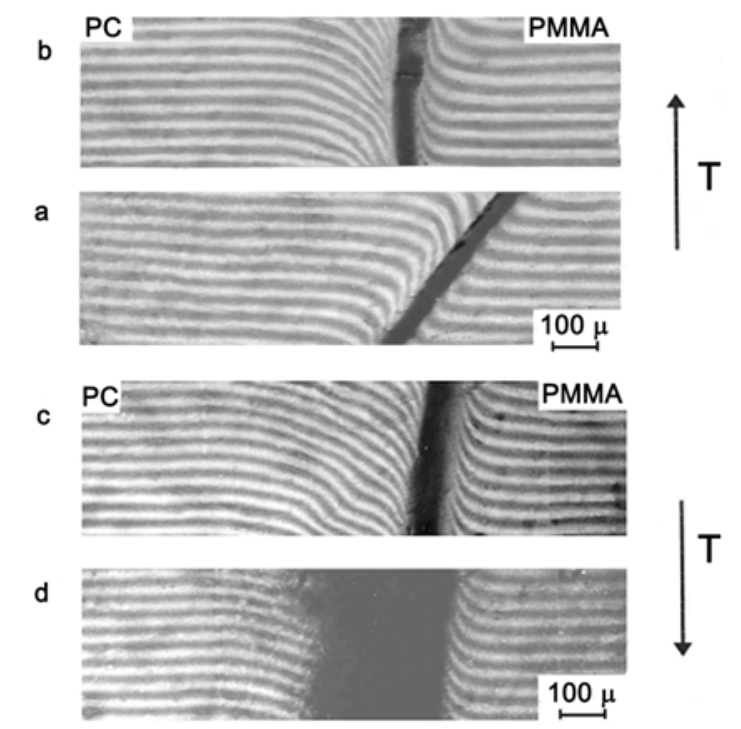

Fig. 6. Interferograms of PC-PMMA $\left(\mathrm{Mw}=1.5 \cdot 10^{4}\right)$ system obtained at increase of temperature from 200 (a) to $260^{\circ} \mathrm{C}$ (b) and at decrease of temperature from 260 to 220 (c) and $160^{\circ} \mathrm{C}$ (d). 
At cooling the compatibility decreases and in the solutions domain close to the interface the concentration of one component becomes higher than thermodynamically equilibrium (super-saturation) that causes appearance of the disperse phase enriched by this component. Due to high dispersity degree this two-phase region scatters light intensively and it looks more dark than single-phase regions. With time on boundaries between homophase and heterophase domains the concentrations corresponding to spinodal branches are realized (Fig 5, curve $3^{*}$ ) and bending of interference bands decreases (Fig. $6 \mathrm{~d}$ ). The concentration width of the meta-stable region located between binodal and spinodal monotonously decreases at moving from low to higher temperatures: from $8 \%$ at $190^{\circ} \mathrm{C}$ to $2 \%$ at $270{ }^{\circ} \mathrm{C}$ from the side of the higher content of PC of and from 5 to $2 \%$ at the same temperatures from the side of PMMA.

Since the compatibility of PC and PMMA of higher molecular weights is very low, the further calculations and experiments on kinetics of dissolution and measuring the viscous properties were carried out for the system PC-PMMA with $\mathrm{M}_{\mathrm{w}}=1 \cdot 5 \cdot 10^{4}$.

For the quantitative interpretation of experimental data on interdiffusion the theory of Flory-Huggins-Scott (FHS) (Scott, 1949; Tompa, 1956) was applied. Partially, from classical expression for the Gibbs free energy the interaction parameter $\chi$ was calculated. Calculation of interaction parameter was performed assuming its linear dependence on concentration (Koningsweld\& Kleintjens, 1977; Palatnik\&Landau, 1961):

$$
\chi=\chi_{0}+\alpha \varphi_{1}
$$

where $\chi_{0}$ - the interaction parameter at $\varphi_{1}=0, \alpha$ - the constant, reflecting influence of blend composition on intermolecular interaction.

Based on concentrations corresponding to binodal branches at definite temperatures, the values of $\chi_{0}$ and $\alpha$ were calculated using FHS equation and the expression (1). The family of linear dependences $\chi\left(\varphi_{1}\right)$ for different temperatures is shown in Fig. 7. The calculated values of the interaction parameter $\chi$ lie in limits of $0.017-0.030$, that is inherent for good compatibility of components. At moving from PMMA to PC, the $\chi$ value decreases that confirms higher solubility of PMMA in PC than vice versa.

At relatively low temperatures the concentration dependence of the interaction parameter $\chi$ is strong enough (the value $\alpha$ in Eq. (1) at $212^{\circ} \mathrm{C}$ is equal to 0.004 ), but it decreases with increase of temperature reaching the constant level 0.002 (Fig. 8). Using this fact as well as zero-meaning of the second and the third derivatives in critical point, the expression for $\varphi$ and $\chi$ at UCMT was obtained (the dependence $\varphi(x)$ was also taken into account):

$$
\begin{gathered}
\varphi_{2 \kappa p}=\frac{\sqrt{\frac{x_{1}}{x_{2}}-6 \alpha x_{1}}}{1+\sqrt{\frac{x_{1}}{x_{2}}-6 \alpha x_{1}}} \\
\chi_{12}=\frac{1}{2}\left(\frac{1}{\sqrt{x_{2}}}+\frac{1}{\sqrt{x_{1}-6 \alpha x_{1}}}\right)^{2}+\alpha\left(5 \varphi_{2 \kappa p}-1\right)
\end{gathered}
$$


These equations allowed us to calculate critical values: $\varphi_{c r}=0,61, \chi_{c r}=0,017$ and by extrapolation of $\chi$ at $\varphi=\varphi_{c r}$ to $\chi_{c r}$, determine the critical temperature equal to $296^{\circ} \mathrm{C}$. The calculated value of UCMT allowed us to plot the complete phase diagram of this system.

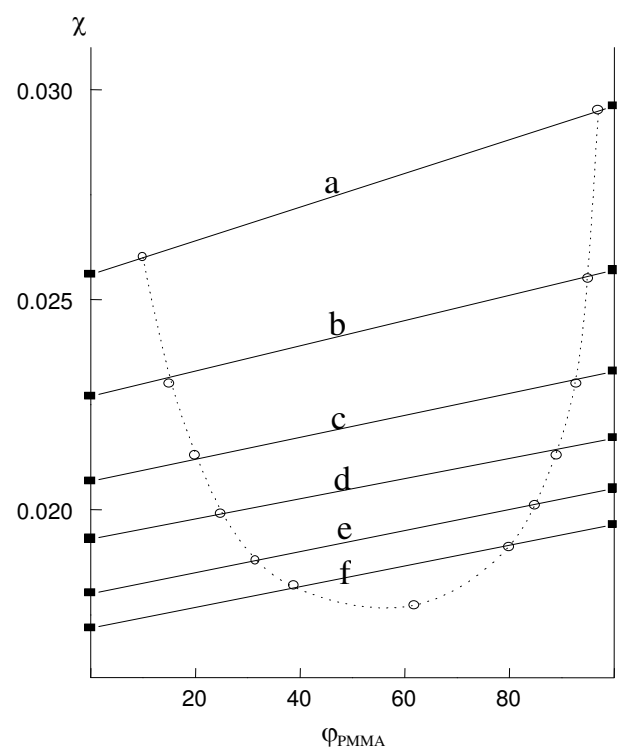

Fig. 7. Concentration dependence of the interaction parameter for the system PC-PMMA $\left(\mathrm{M}_{\mathrm{w}}=1.5 \cdot 10^{4}\right) . \mathrm{T}=212(\mathrm{a}), 226$ (b), 240 (c), 254 (d), 268(e) и 282。C (f). The dotted line corresponds to the $\chi$ values on the binodal.

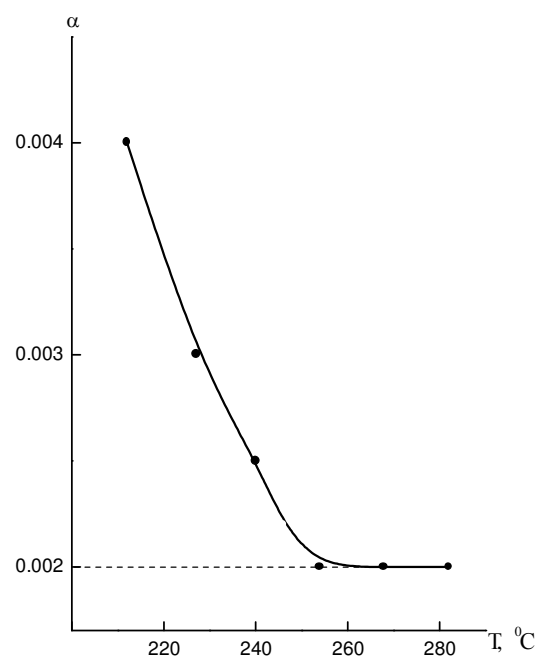

Fig. 8. Temperature dependence of the $\alpha$ constant in Eq. (1) for the same system. 
The $\varphi_{c r}$ value (Fig. 5, point $a$ ) calculated with taking into account the concentration dependence of the interaction parameter differs almost on $10 \%$ on $\varphi_{c r}$ value estimated if this dependence is ignored (Fig. 5, point $b$ ) (Koningsweld\& Kleintjens, 1977; Palatnik\&Landau, 1961). This means that the critical concentration depends not only on dimensions of macromolecules, but on their nature as well.

For description of kinetics of mixing the interdiffusion coefficients $D_{v}$, characterizing the rate of interpenetration of components, were calculated. The calculation was done by graphic method from the curves of concentration distribution by means of MatanoBoltzman method (Borovskii, 1973) at three temperatures and concentration domains of compatibility (Fig. 10). Interdiffusion coefficients are rather high for polymer pairs and change from $1,5 \cdot 10^{-12}\left(220^{\circ} \mathrm{C}\right)$ to $4 \cdot 10^{-11} \mathrm{~m}^{2} / \mathrm{c}\left(260^{\circ} \mathrm{C}\right)$. $\mathrm{D}_{\mathrm{v}}$ values in the region of predominant PMMA content are a little lower than for the region of higher PC content.

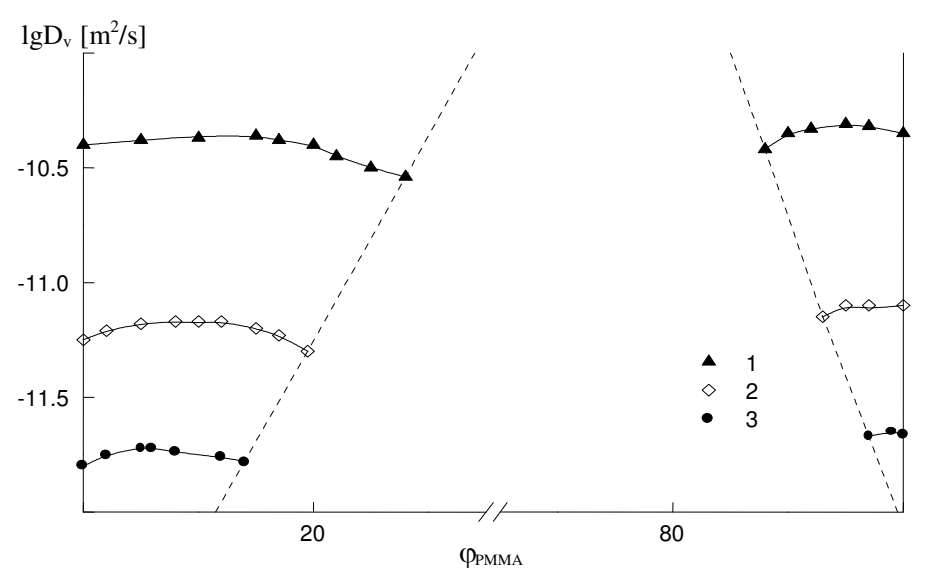

Fig. 9. Interdiffusion coefficients vs. composition for PC-PMMA $\left(\mathrm{M}_{\mathrm{w}}=1.5 \cdot 10^{4}\right)$ system at 260 (1), 240 (2) and $220^{\circ} \mathrm{C}$ (3). The domain restricted with dotted line corresponds to the biphasic region of the phase diagram.

The concentration dependence of the interdiffusion coefficient far from binodal is rather weak. With increase of the one component content in another $D_{v}$ values a little increase but at approaching to binodal decrease again. Deceleration of diffusion process in vicinity of saturation state for both solutions is natural, since $D_{v}$ is proportional to derivative of chemical potential on concentration that becomes equal to zero on the spinodal.

With increase of temperature interdiffusion coefficients become higher for all compositions (Fig. 9). Activation energy of diffusion is high enough for polymer systems and equal to 170 $\mathrm{kJ} /$ mole. Extrapolation of the temperature dependence of $\mathrm{D}_{\mathrm{v}}$ to $160^{\circ} \mathrm{C}$ gives a value of $8.1 \cdot 10^{-}$ $15 \mathrm{~m}^{2} / \mathrm{c}$. Probably, the high difference in diffusion mobility of components at high (4:10-11 $\mathrm{m}^{2} / \mathrm{c}$ at $260^{\circ} \mathrm{C}$ ) and low temperatures close to the glass point could be the explanation of false LCST's [14-19]. This situation can be realized in attempts to create the homophase 
mixed solutions of PC and PMMA in a common solvent. After removing a solvent the thermodynamically unstable system at heating suffers very slow noticible decomposition at $T^{*}>T_{g}$ only, because of low $D_{v}$ values. The $T^{*}$ value could be accepted as LCST.

One of the practical application of obtained data on interdiffusion coefficients and mutual solubilities of partially compatible components consists in estimation of time needed for formation of the interphase layer of the definite thickness that should influence on properties of the heterophase system including rheological and mechanical ones. A volume of the interphase layer was accepted as the tenth part of disperse particle volume. Calculation was done using the model of dissolution of a particle of spherical shape (Crank, 1956):

$$
\frac{\varphi}{\varphi_{0}}=\frac{a}{r} \operatorname{erfc} \frac{r-a}{2 \sqrt{D_{v} \tau}}
$$

where $\varphi$ and $\varphi_{0}$-concentrations of the component on the inner and the outer boundaries of the interphase layer, $a$ - a radius of a particle, $r$ - a distance from particle center to the border of the layer, erfc - empirical probability function (taken from the reference book). The PMMA was chosen as the disperse phase and PC as the disperse medium. The concentration of PC onto interface was accepted as in $e$ times less than the initial concentration. Results of the calculation for $240^{\circ} \mathrm{C}$ are presented in Table 1 . One can see that time of spontaneous formation of such a layer depends strongly on the particle dimensions and can differ from a few minutes to several years. This difference underlines again the determining role of dispersity at dissolution of polymers.

\begin{tabular}{|c|c|c|}
\hline $\begin{array}{c}\text { Thickness of the } \\
\text { diffusion layer, } \mu\end{array}$ & $\begin{array}{c}\text { Diameter of a } \\
\text { particle, } \mu\end{array}$ & $\begin{array}{c}\text { Time of formation of the diffusion layer of } \\
\text { the definite thickness }\end{array}$ \\
\hline 0,3 & 10 & 5 min \\
\hline 3 & 100 & $8,3 \mathrm{~h}$ \\
\hline 15 & 500 & 8,7 days \\
\hline 30 & 1000 & 34,7 days \\
\hline 60 & 2000 & 4,6 months \\
\hline 150 & 5000 & 2,4 year \\
\hline
\end{tabular}

Table 1. Time of the diffusion layer formation at mixing of PC with PMMA $\left(\mathrm{M}_{\mathrm{w}}=1.5 \cdot 10^{4}\right)$.

\begin{tabular}{|l|c|c|}
\hline \multicolumn{1}{|c|}{ Diameter of a particle, $\mu$} & $\mathrm{T}=240^{\circ} \mathrm{C}$ & $\mathrm{T}=160^{\circ} \mathrm{C}$ \\
\hline 1 & $10 \mathrm{~s}$ & $30 \mathrm{~h}$ \\
\hline 10 & $20 \mathrm{~min}$ & 4 months \\
\hline 100 & $30 \mathrm{~h}$ & 30 years \\
\hline
\end{tabular}

Table 2. Time of complete dissolution of the PMMA particle in PC.

The time of total dissolution of PMMA particle in PC was estimated as well. For comparison, calculations were performed for two temperatures: 160 and 240 $\mathrm{C}$. Results shown in Table 2, illustrate significant delay of interpenetration process at approaching to the glass point. Simultaneously, these data allow us to make choice for the mixing regime to obtain either homogeneous solution of PMMA in PC or three-phase system consisting 
of PMMA, PC and solution of PMMA in PC (as the interphase layer of the definite thickness).

Data on kinetics (interdiffusion coefficients) and thermodynamics (phase equiblibria) of polymers under investigation have been compared with rheological results obtained by squeeze flow method (Kotomin\&Kulichikhin, 1996) - the concentration dependence of the Newtonian viscosity, presented in Fig. 10. The matter is that in both processes (diffusion and flow) a segmental motion takes place. In the case of diffusion it is stipulated by difference of chemical potentials, and in the case of rheology - applying the external force. That is why segments participating in the diffusion and the rheology can be different, but mechanisms are similar (Malkin\&Chalykh, 1979), and joint consideration of data of both methods are undoubtedly valuable.

The concentration dependence of viscosity of PC-PMMA $\left(\mathrm{M}_{\mathrm{w}}=1.5 \cdot 10^{4}\right)$ system at $240 \mathrm{\circ}$ at majority compositions excluding specific domain is located higher of the logarithmic additivity line that indicates on active interaction of components (Kuleznev, 1980). At small content of PMMA the characteristic minimum was observed. At comparing with the phase diagram it is evident that its position is nearst binodal. The viscosity decrease can be explained by appearance of microemulsion with high fraction of interfacial layer with lower density compared with neat polymer melts (Kuleznev, 1980; Kuleznev\&Kandyrin, 2000; Kuleznev, 1987). Presumably, this minimum is connected with meta-stable state of the system where a great number of heterophase fluctuations exists.

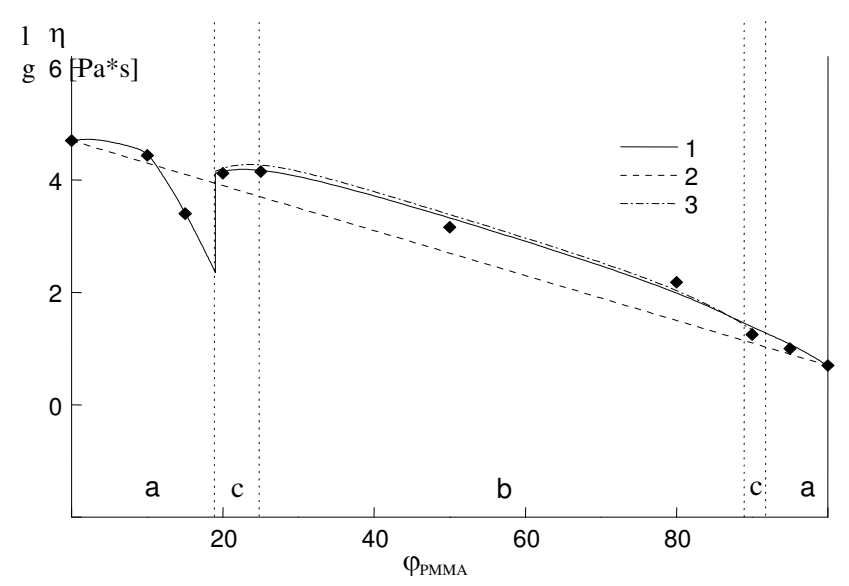

Fig. 10. Viscosity vs. composition curves for the system PC - PMMA ( $\left.\mathrm{M}_{\mathrm{w}}=1 \cdot 5 \cdot 10^{4}\right): 1$ experimental curve; 2 - line of logarithmic additivity; 3 - the curve, calculated using formulae (5). Vertical lines designate domains of solutions (a), meta-stable state (b) and biphasic (c).

The concentration dependence of viscosity in biphasic region was analyzed based on knowledge of the phase diagram. For this aim the empirical formulae was used (Kuleznev\&Kandyrin, 2000): 


$$
\lg \eta=\psi_{2}^{2} \lg \eta_{2}+\left(1-\psi_{2}^{2}\right) \lg \eta_{1}
$$

where $\eta_{1}$ and $\eta_{2}$ - viscosities of disperse phase and medium, respectively, $\psi_{2}$ - the volume fraction of dispersion medium that for compatible polymers coincides with relative content of the component forming the medium. But if at least partial mutual dissolution proceeds, this coincidence is absent. It is why the $\psi_{2}$ value was found from the phase diagram using the meanings of concentrations on binodal and "the lever rule". The calculated curve 3 (Fig. 11) coincides in limits of equipment error with the experimental one.

So, the couple PC - low molecular weight PMMA is a good example of polymer systems with amorphous phase equilibrium. Method of interferometry is very useful and fruitful for such kind of systems due to informativity and combination of joint approaches: kinetics of mass-transfer and thermodynamics of phase states. Increase of the mutual solubility with temperature indicates on UCST that could not be measured experimentally but was calculated using evolution of the interaction parameter with concentration.

Comparison of the phase diagram with concentration dependence of viscosity allowed us to observe local minimum of viscosity near the binodal (sooner in meta-stable region between binodal and spinodal where microemulsion appears as the first step of the phase decomposition) and to apply the empiric equation for calculation of the blends viscosity by means of accurate determination of the composition from binodal branches. Joint consideration of diffusion and rheological properties seems to be very fruitful for judging about structure, kinetic and thermodynamic behaviors of complex, multiphase polymer systems. This approach will be used further for analysis of systems with LC and crystalline equilibria.

Calculations of the interphase layers thickness depending on dimensions of drops and time, based on the knowledge of interdiffusion coefficients can be very useful for correct organization of technological process of mixing and, consequently, development of new materials with three-phase structure and new properties.

\section{Crystalline and complex phase equilibria in polymer systems}

Crystalline equilibrium is inherent for polymer systems if at least one component is capable to crystallize. At cooling from the region of the total compatibility (isotropic solution) at definite temperature the system decomposes on two equilibrium phases: deposition of the crystalline component and its saturated solution into the other component.

The curve describing sum of transition (melting or crystallization) points corresponding to various compositions is named "liquidus". Higher of the liquidus line the single-phase region (liquid solution) exists, and lower the liquidus line - biphasic system (crystalline phase distributed in solution). Basic kinds of phase diagrams with crystalline equilibrium are shown in Fig. 11.

Cases a) and b) are typical for components with a weal interaction. If one component of such a system is amorphous and the other - crystalline, the melting point of a system as whole decreases monotonously as a result of dilution of the crystalline component by amorphous one (depression of melting points) as is seen in the graph a). In systems containing two components capable to form crystalline phases two liquidus line cross in the eutectic point 

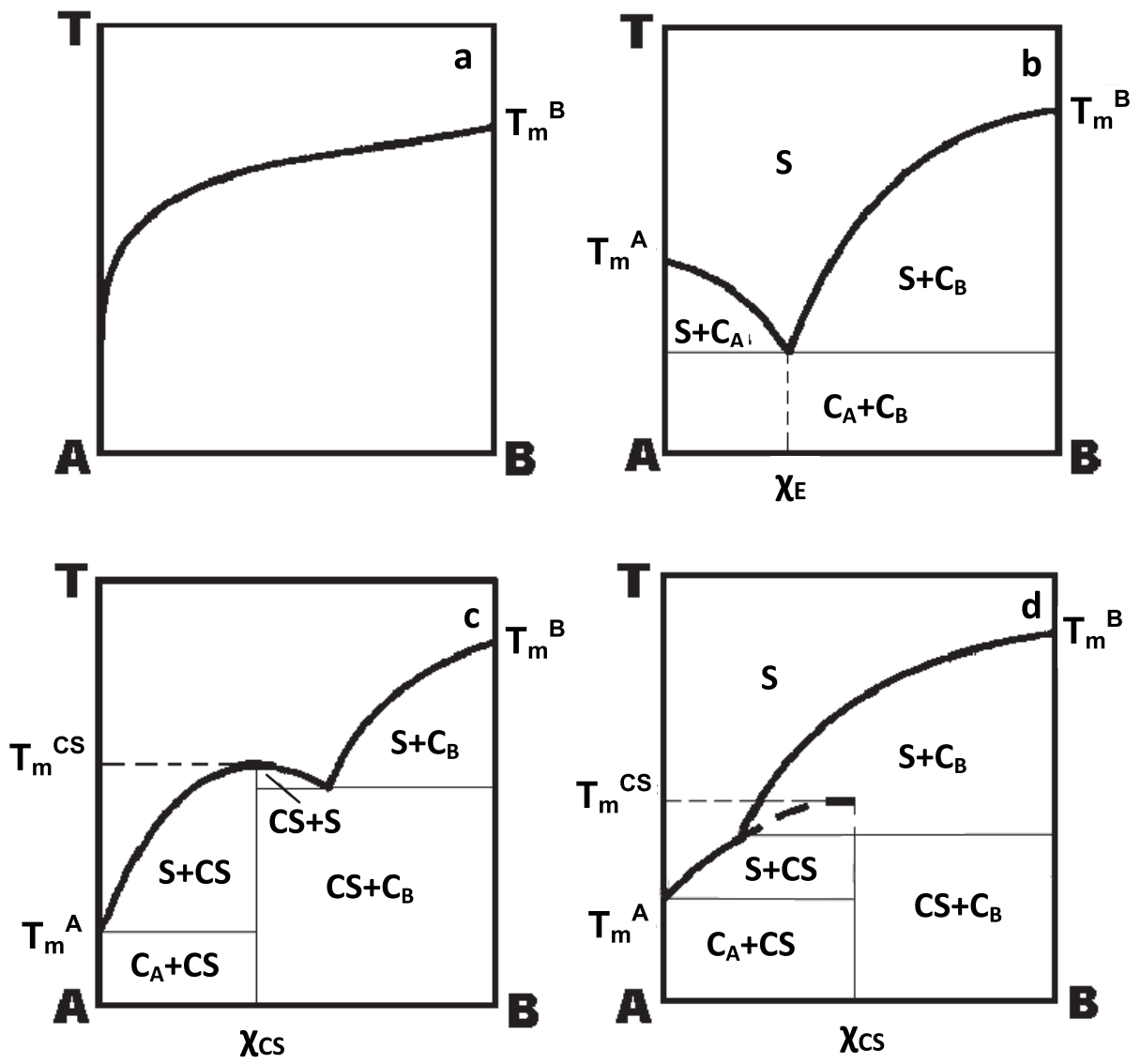

Fig. 11. Basic types of phase diagrams with crystalline equilibrium $[5,6,9]$.

located in position lower than melting points of neat components (type b). Under the left liquidus curve up to $x_{e}$ point the equilibrium of the crystalline phase $\mathrm{A}$ and its saturated solution exists, and after $x_{e}$ - the equilibrium of the crystalline phase B with its saturated solution. In the temperature region lower of the eutectic point the biphasic domain consists of crystals A and B only.

Phase equilibria of types c) and d) are inherent for systems with polar components and their interaction leads to formation of crystalline additive compounds - crystal-solvates (CS). At congruent melting of CS (without its decomposition on components) the maximum melting point $\mathrm{T}_{\mathrm{m}} \mathrm{CS}$ exists (type $\mathrm{c}$ ). But if the energy of the additive compound formation is low, (incongruent melting) its decomposition takes place until reaching $\mathrm{T}_{\mathrm{m}}{ }^{\mathrm{CS}}$ (type $\mathrm{d}$ ). Under liquidus curve in region of compositions less than $x_{C S}$ there exist in equilibrium phases of CS of $x_{C S}$ composition and saturated solution (S) B in A $\left(T>T_{m}{ }^{A}\right)$ or crystals $(C)$ A $\left(T<\mathrm{T}_{m}{ }^{A}\right)$. Passing through $x_{C S}$ in the temperature region lower the eutectic point, the CS phase coexists with saturated solution of A in B. 
The maximum number of phase diagrams for polymer systems with crystalline equilibrium is devoted to polymer-solvent systems (Papkov, 1971; Tager, 1978; Papkov, 1981). One of the classical examples is the phase diagram of poly-p-phenyleneterephthalamide (PPTA) $\mathrm{H}_{2} \mathrm{SO}_{4}$ schematically shown in Fig. 12. (Iovleva\&Papkov, 1982; Papkov et al., 1986; Kulichikhin, 1989). But in the particular case of stiff-chain polymers solutions the LC phase appears at definite concentration of polymer separated from isotropic solutions by narrow biphasic "corridor". The situation with LC equilibrium will be considered later, but we should indicate here on the right part of the diagram where formation of the CS phase with congruent melting is realized. In this case the knowledge of the phase diagram was used for practical aim of choosing the most appropriate concentration of dopes and the temperature regime of spinning the super-strong fibers of Kevlar (Terlon) type (Papkov\&Kulichikhin, 1977).

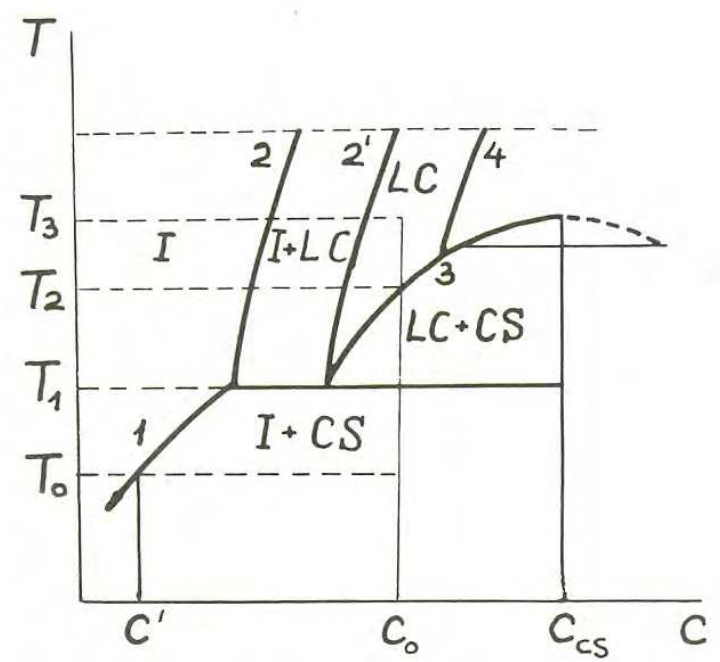

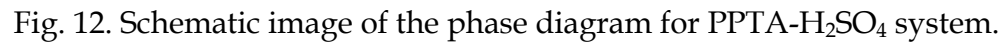

Coming back to polymer-polymer interaction let us consider now several binary systems based on polyesters. These polymers are of interest from the viewpoint of mixing just to reach the new complex of properties hoping at least on partial physical compatibility. Simultaneously, we should keep in mind a possibility of chemical interaction of polyesters via mechanisms of acidolysis or alkoholysis which involve ester-ester exchange. So, in this case interdiffusion can be stipulated as chemical similarity as chemical interaction leading to formation of copolyesters. Let us accept that $280-290{ }^{\circ} \mathrm{C}$ is the border temperature that separates the low-temperature region where physical interaction (diffusion) prevails, and high-temperature region where chemical interaction becomes significant.

\subsection{Interaction between LC- and isotropic polyesters melts}

At low-temperature region two couples of polyesters have been studied. In the first one two components were in interaction: polydecamethyleneterephthaloil-bis-4-hydroxybenzoate LC polyester and polybutyleneterephthalate (PBT) (Kulichikhin et al., 1994). PBT is semi- 
crystalline polymer. This pair has some specific features from the viewpoint of application of method of the optical interferometry. In the case of LC polyester-PBT couple the melt of the first polymer has LC ordering up to clearing (isotropization) point. This means that LC melt is not transparent that is why the evolution of interference bands during interaction of two above indicated melts was controlled from one side - transparent PBT melt only.

At room temperature both polymers are non-transparent. At $230^{\circ} \mathrm{C}$ PBT melts and intrinsic for it interference bands appear while LC polyester even in melt state remains dark. Bending of PBT interference bands in vicinity of the interface indicates on dissolution of LC polyester in PBT, and in concentration profile gently sloping section is seen. At T $290^{\circ} \mathrm{C}$ LC polyester becomes isotropic and the interference picture indicates on complete compatibility of components in these conditions. The inereference data allowed us to construct the phase diagram (Fig. 13).

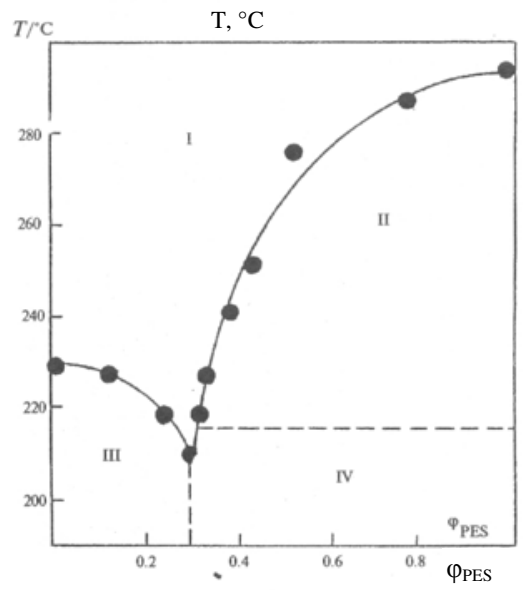

Fig. 13. Phase diagram for the system LC polyester - PBT. I - isotropic solutions, II - LC state of LC polyester solutions in PBT, III crystalline state of PBT saturated solutions, IV crystalline state of LC polyester.

There are two liquidus lines with eutectics at volume fraction of PBT=0.7. The presence of eutectics confirms by preservation of transparent zone for this composition up to $170^{\circ} \mathrm{C}$, i.e. much lower of crystallization points of neat components and solutions of other compositions (Fig. 14). In heating cycle the eutectic zone is clearing the first and then zones of other compositions. This situation is very similar to the classical case of crystalline equilibrium expressed by pattern b) in Fig. 11.

The second system was a pair of polyehyleneterephthalate (PET) and LC copolyester of PET with p-hydroxybenzoic acid (PET/HBA) one half of the interference picture is dark, but sloping the interference bands of transparent PET near interface indicates on partial dissolution of PET/HBA anisotropic melt in PET at $290^{\circ} \mathrm{C}$ forming interphase layer with a thickness increasing in time. Unfortunately, the clearing point of PET/HBA copolyester is higher than $350^{\circ} \mathrm{C}$ and higher than decomposition point of this polymer. That is why we cannot say definitely about degree of compatibility, but an increase of bending is observed 


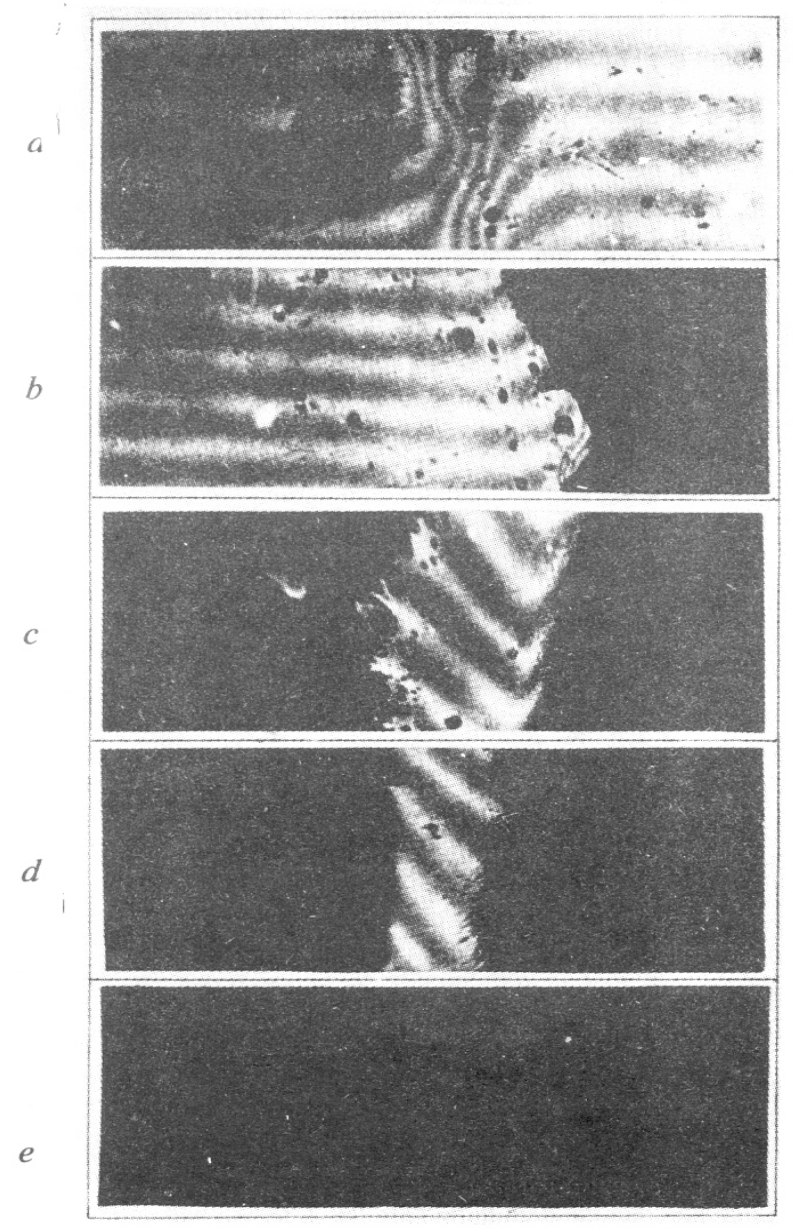

Fig. 14. Evolution of the interference patterns in the diffusion zone at cooling the system LC polyester - PBT. Temperature: 310 (a), 270 (b, LC polyester crystallizes), 220 (c, PBT crystallizes), 210 (d, the eutectic zone is lighted up), and $20^{\circ} \mathrm{C}(\mathrm{e})$.

that indicates on continuation of diffusion interaction in this time interval (Tereshin at al., 2000). So, in both cases the method of observation on one half of the interference pattern related with transparent polymer melt was developed. This approach could be very useful for analysis of diffusion interaction between two polymers, of one of them is turbid.

So, in previous cases the experiments on optical interferometry were carried out up to temperature of $290^{\circ} \mathrm{C}$. Presumably, this is lower than it is possible to expect active chemical interaction between two polyesters, especially if one of them is LCP. The matter is that due to orientation of LC domains onto interface and formation more dense boundary layer, both processes - diffusion and chemical interaction can be slowed. For isotropic polyesters the situation with physical and chemical interactions should be more clear. 


\subsection{Pair of PET with polyethylenenaphtalate (PEN)}

In the case of PET-PEN pair a special attention was devoted to superposition of two processes: interdiffusion and typical for polyesters interchain chemical reaction of the esterester exchange. These components are miscible completely, finally forming copolyester, and the main problem consisted in comparison of diffusion and chemical reaction rates. In our opinion, the first stage of interaction of molten polyesters is interdiffusion, and the second one - chemical trans-esterification. Transition from one stage to another is reflected on kinetics of concentration profile suffering significant change that time.

PET is a large-capacity thermoplastic used for the production of fibers, films, engineering plastics, and packaging articles. PET articles demonstrate excellent mechanical properties, with their manufacture technique being relatively simple. Nevertheless, some properties of PET do not suit consumers. Specifically, this refers to the phase state of PET, which belongs to the family of semi-crystalline polymers and possesses a high crystallinity degree (40$45 \%)$. Crystallites resulting from the rapid cooling of thin-walled articles are too small in size to affect the optical properties of PET articles. However, in the case of bulky thickwalled products, crystalline structures forming in the course of cooling lead to the loss of transparency. As a consequence, the scope of potential applications of PET appears to be limited. Moreover, a relatively low heat resistance (the glass transition temperature of PET is $\sim 80^{\circ} \mathrm{C}$ ) provides no way of using PET articles at high temperatures.

It is advisable to use as a partner for PET poly(ethylenenaphthalate) (PEN) both for physical (interdiffusion at relatively low melt temperatures) and chemical interaction (at high temperatures). The most universally adopted mechanism of trans-esterification relies on the decisive role of hydroxyl end terminal groups that participate in alcoholysis and acidolysis reactions and enter to neighboring chains of polyesters (Fakirov, 1999). The block copolymers thus produced improve the compatibility of polyesters with different chemical structures. Subsequently, neighboring polyester chains undergo scission and recombination reactions (direct ester-ester exchange), making copolymers random in structure (Stewart et al., 1993).

Over many years, it has been agreed that this reaction is responsible for compatibility of blend components (Litmanovich et al., 2002; Ihm et al., 1996). However, Guo\&Brittain, 1998, revealed that trans-esterification is not prerequisite to compatibility. According to solid-state NMR spectroscopy, a mixture of melts became compatible during the first $1.5 \mathrm{~min}$ of injection molding and only after that could signs of the trans-esterification reaction be detected. The authors of the cited work proposed that, for the chemical interaction to occur, chains should be situated close to each other (at least within the action of dispersion forces). It is natural that for the incompatible blends of molten polyesters, the existence of interfaces limits coming together of potentially reactive macromolecules.

Thus, polymers making up the blend should form single-phase regions so that the effective chemical interaction can take place. Okamato\&Kotaka, 1997, have demonstrated that the rapid cooling (quenching) of a PET/PEN blend being extruded at $280^{\circ} \mathrm{C}$ leads to the heterophase melt morphology (probably, because of spinodal phase decomposition). Further melting and annealing initiate the occurrence of trans-esterification at the boundaries of domains and their gradual disappearance as a result of compatibility. The use of a random PET-PEN copolymer as a third component accelerates compatibility. 
Analogous conclusions concerning the incompatibility of the physical blend and the decisive role of trans-esterification in the compatibility of PET and PEN were reported in (Ihm et al., 1996). If the degree of interchain exchange is greater than $50 \%$, blends do not crystallize and a single glass transition point is observed only. The rate of trans-esterification proves to be temperature- and time-dependent; however, it is unaffected by the blend composition.

Thus, the above seemingly simple and evident approach to modifying the properties of PET, which involves its blending with a certain amount of PEN, is complicated by the chemical interaction of these two polyesters. However, kinetics of physical and chemical processes and its determining factors, allowing us to estimate all stages of interaction are still unknown completely. The compatibility of molten PET and PEN calls for further investigation, and in this Chapter we focus our attention just on this problem (Makarova, 2005).

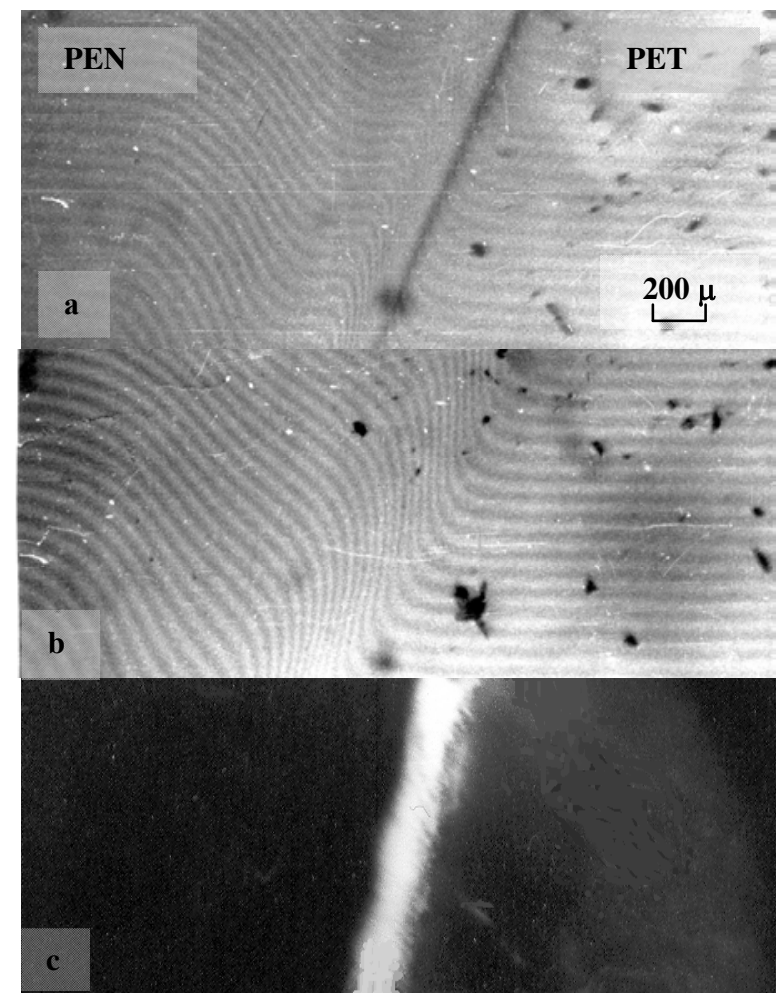

Fig. 15. Interferograms of PET - PEN system at $290^{\circ} \mathrm{C}$ in 5 (a) and $10 \mathrm{~min}$ (b) after contact. The pattern (b) corresponds to the combined specimen cooled to ambient temperature from $290^{\circ} \mathrm{C}$.

At contacting PET and PEN after the melting of PET $\left(\sim 260^{\circ} \mathrm{C}\right)$ the weak bending of the interference bands occur that is caused by penetration to PET of macromolecules of still crystalline PEN $\left(\mathrm{T}_{\mathrm{m}}=266^{\circ} \mathrm{C}\right)$. A solubility of PEN in PET in such conditions is not more 5$10 \%$. After PEN melting a lot of sloped bands appear from both sides of the interface, and 
number of them increases with time (Fig. 15a). In $10 \mathrm{~min}$ (this time depends on temperature) the interface disappears completely (Fig. 15b). After cooling the cell to ambient temperature the region in vicinity of the former interface remains light (Fig. 15c). Presumably, either amorphous solution of components is formed in this region (eutectic point) or amorphous transparent copolyester.

Presence of the definite time lag (or "time barrier") as well continuous increasing a number of interference bands in isothermal conditions is not typical for the case of the physical compatibility, since at full compatibility the interface is absent already at first contacting of components. So, such behavior can be caused by simultaneous passage of interdiffusion and chemical interaction, promoting compatibility of components in time.

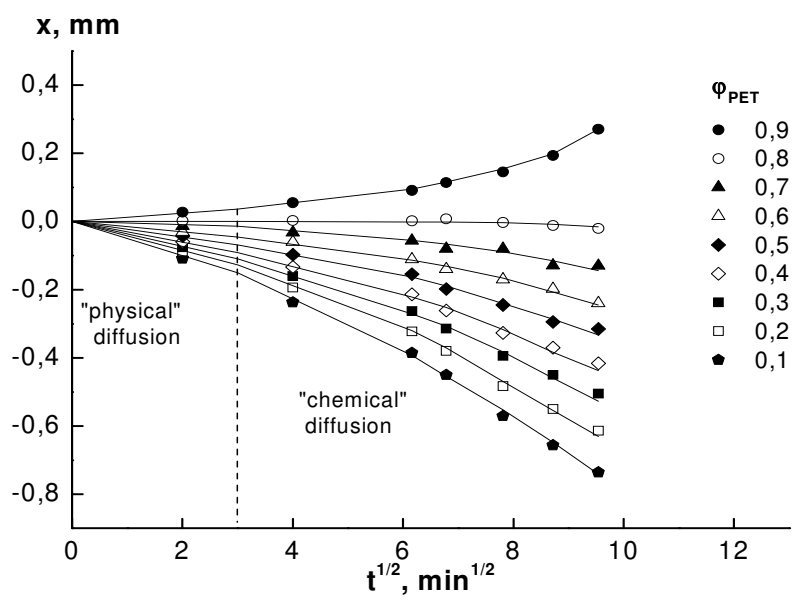

Fig. 16. Movement of iso-concentration fronts for the system PET - PEN at $300^{\circ} \mathrm{C}$.

Plotted on the base of interferograms, concentration profiles in the transient zone are asymmetric from the PEN side that witnesses about prevail penetration of PET into PEN. Movement of the iso-concentration plains $x(\varphi)$ calculated from concentration profiles, follows to diffusion laws $\left(x \sim t^{0.5}\right)$ on the initial sections only (Fig. 16) which length does not exceed $10 \mathrm{~min}$. This time is comparable with disappearance of the interface. The further nonlinearity can be connected with chemical interaction. In the figure this stage is noted as "chemical diffusion" because traditional diffusion rules do not work for chemically different elementary units.

For analysis of the difference in behavior of copolymers and complementary blends the interdiffusion was investigated on specimens consisting of three components: PET, PEN and copolyester or blend of the same composition. Copolyesters were synthesized via chemical reaction of PET with 2,6-hydroxynaphthoic acid (HNA) (Makarova et al, 2005). For the combined specimen "PET-PEN-copolyester" the interferograms are shown in Fig. 17. After PEN melting the interface is presented only from the PET side, while from copolyester side the continuous concentration profile is observed. Probably, the above discussed "time lag" is stipulated by necessity of copolyesters formation accelerating the further compatibility. 

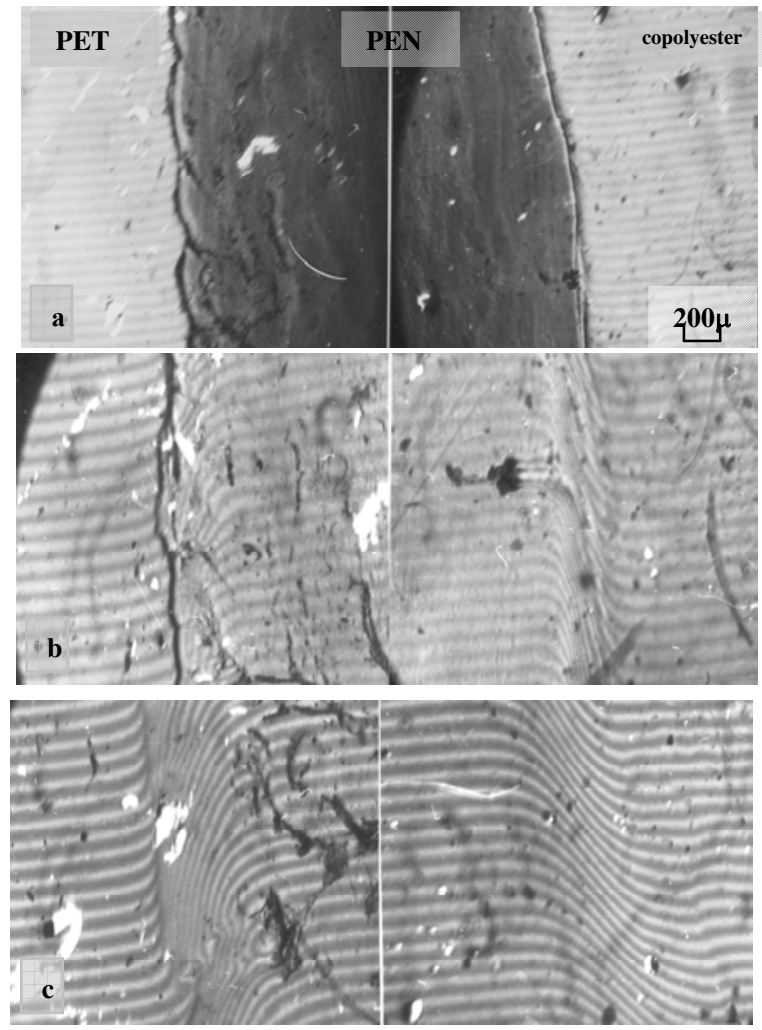

Fig. 17. Interferograms of PET - PEN - copolyester PET/HBA=80/20 at 268 (a) and $272^{\circ} \mathrm{C}$ in 10 (b) and $30 \mathrm{~min}(\mathrm{c})$.

Combination of PEN with mechanical blend PET/PEN=90/10 and copolyester containing $10 \%$ of HNA at $280^{\circ} \mathrm{C}$ (Fig. 18) has shown an absence of interfaces from both sides. However at duration of observation $20 \mathrm{~min}$ the bending of interference bands preserves from a side of the blend. Disappearance of such bending from a side of the copolyester indicates on fast redistribution of concentrations in this diffusion zone.

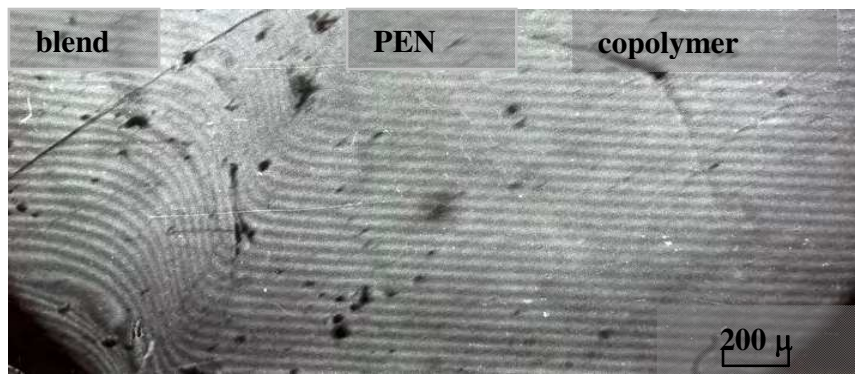

Fig. 18. Interferogram of the combined system: mechanical blend PET/PEN=90/10 - PEN copolymer $\mathrm{PET} / \mathrm{HNA}=90 / 10$ at $280^{\circ} \mathrm{C}$ in $20 \mathrm{~min}$ after contact. 
In some cases, at slow cooling as PET itself as the combined specimen PET - PEN the formation of tree-like morphology was observed similar to fragments of dendrite spherolites. "Branches" of PET were more monolithic and dense compared with graceful branches formed in PET containing 5\% of PEN (Fig. 19). This fact can be explained by decrease of crystallinity degree of PET at adding minor amount of PEN and formation of amorphous copolyester. In this circumstances PET crystallites can grow and form spherolites.

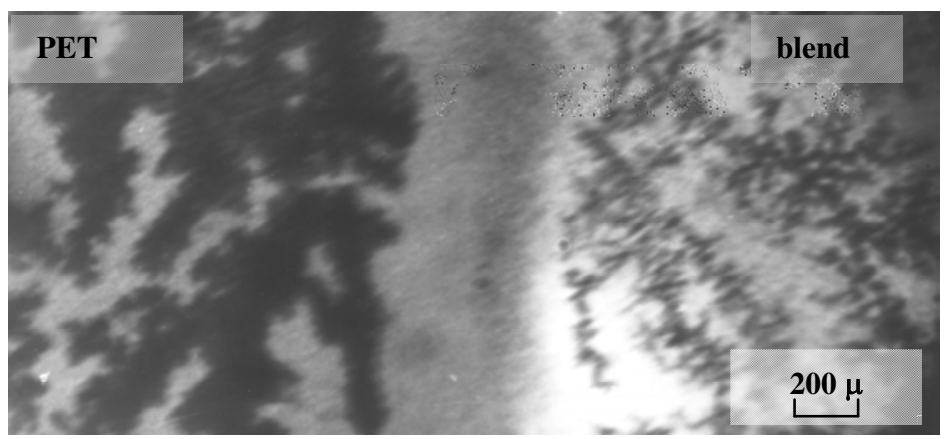

Fig. 19. Micrograph of the diffusion zone at contact of PET with mechanical blend PET/5\% of PEN.

Using concentration profiles in the diffusion zone PET-PEN, interdiffusion coefficients were calculated at different temperatures. The $\mathrm{D}_{\mathrm{v}}$ values in this temperature range lie in diapason $1.4 \cdot 10^{-12} \div 5.4 \cdot 10^{-11} \mathrm{~m}^{2} / \mathrm{s}$. The apparent activation energy of the diffusion process is closed to $410 \mathrm{~kJ} /$ mole. It is likely that so high value of the temperature coefficient can be explained by additional input of trans-esterification into the process of mutual penetration of components. Since dependence of $D_{v}$ on concentration is rather weak, further calculations for various compositions at one temperature were carried out assuming independence of $D_{v}$ on composition. The algorithm of calculations was the same as for PC-PMMA system using the probability diagram (Crank, 1956; Avdeev, 1990).

Calculated values of $D_{v}$ initial polyesters and their combination with mechanical blends and copolyesters of complementary compositions are presented in Table 3. These data reflect more fast interpenetration of homo- and copolyesters compared with blends. The corresponding $\mathrm{D}_{\mathrm{v}}$ values differ in 2-10 times.

It is rather strange that at contact of homopolyesters with mechanical blends the diffusion penetration is less intensive even than for homopolymers. Since blends were obtained at temperatures higher $280^{\circ} \mathrm{C}$ during $10 \mathrm{~min}$ and more, it is reasonable to expect the definite fraction of copolyesters formation. Nevertheless their interaction with homopolymers is not too intensive as for pure copolyesters. Probably, this can be explained by chaotic morphology of blends that leads to formation of heterogeneous interfaces and to interaction with homopolymer melts different species. So, presence in the mixture of copolyesters not ever accelerates the interpenetration. They should be just at interface as in the case of interaction of homo- and copolyesters.

The hypothetic mechanism of the general interaction in polyesters should be the following. First of all, the interdiffusion between components proceeds resulting in interpenetration of 


\begin{tabular}{|l|c|}
\hline \multicolumn{1}{|c|}{ Systems } & $\mathrm{Dv}_{\mathrm{v}} \cdot 10^{12}, \mathrm{M}^{2} / \mathrm{c}$ \\
\hline PET - PEN & 2,0 \\
PET - blend PET/PEN=80/20 & 0,2 \\
PET - copolyester PET $/ \mathrm{HNA}=80 / 20$ & 3,0 \\
PEN - blend PET/PEN=90/10 & 3,5 \\
PEN - copolyester PET $/ \mathrm{HBA}=90 / 10$ & 7,0 \\
PEN - blend PET/PEN=80/20 & 0,5 \\
PEN - copolyester PET /HBA=80/20 & 4,5 \\
\hline
\end{tabular}

Table 3. Interdiffusion coefficients for different interacting components at $280^{\circ} \mathrm{C}$.

macromolecules of different sorts. At their closing on a distance permitted for covalent exchange, the chemical process starts leading to formation of copolyesters in vicinity of interface. Further, copolyesters promote as diffusion interaction, as propagation of chemical reaction front in depths of both reacting components.

We can estimate the dissolution time of a particle of PEN or PET in an unrestricted volume of another polymer. Calculations were done in the same way as in the case of PC-PMMA pair (Avdeev, 2001; Makarova, 2007). The corresponding data are presented in Table 4.

\begin{tabular}{|l|l|}
\hline $\mathrm{d}, \mu$ & $\mathrm{T}=280^{\circ} \mathrm{C}$ \\
\hline 1 & $0,1 \mathrm{~s}$ \\
\hline 10 & $10 \mathrm{~s}$ \\
\hline 100 & $20 \mathrm{~min}$ \\
\hline 1000 & $30 \mathrm{~h}$ \\
\hline
\end{tabular}

Table 4. Time of complete dissolution of PEN drop of various size in PET.

These data show that in any industrial mixing method only droplets with a size of $<10 \mu \mathrm{m}$ can be dissolved completely. For larger droplets the interphase layer of the definite thickness will be formed consisting of mutual solutions of homopolymers and copolymers. At cooling the system will contain 3 or 4 phases, influencing its exploring properties.

\section{LC phase equilibrium in polymer systems}

Liquid-crystalline state is stable, thermodynamically equilibrium and engages a gap between amorphous and crystalline states (Papkov et al., 1974; Papkov\&Kulichikhin, 1977; Collyer, 1996; Kulichikhin, 2000]. In LC state macromolecules form uni-dimensional or twodimensional order that distinguishes it from full disorder in amorphous state and threedimensional order in crystalline state. Such state is named often as anisotropic since presence of the order in macromolecular arrangement leads to appearance of anisotropy of many physical properties, partially optical, rheological (majority of these systems are capable to flow) and many others.

LC phase in polymers can be realized either in melt state, if 3D crystalline order disappears not completely, but step-by-step destruction at melting (thermotropic LC polymers) or in 
solutions of stiff-chain polymers (lyotropic LC state). In the last case LC equilibrium takes place at full compatibility of components. Formation of LC solutions is stipulated predominantly by geometric asymmetry of rigid macromolecules which can engage any position in solution volume up to definite concentration only. After critical concentration adding new macromolecule is possible at condition of partial (in the beginning) or full ordering of other macromolecules. Using a term "partial ordering" means that the transition from isotropic to LC state along concentration axis passes via biphasic region where isotropic and LC state coexists.

The general view of the state diagram for solution of stiff-chain polymer with rod-like macromolecules, based on theoretical paper of Flory (Flory, 1956) and Papkov (Papkov et al., 1974; Papkov, 1992) is shown in Fig. 20. The diagram contains a combination of narrow and wide biphasic regions separating isotropic and anisotropic solutions. According to Flory, the critical concentration of LC phase appearance is related with molecular stiffness by equation:

$$
\varphi_{2}^{*} \approx \frac{8}{x}\left(1-\frac{2}{x}\right)
$$

where $x$ is asymmetry degree of macromolecules or, in common case, the ratio of the Kuhn segment to diameter $x=\frac{A}{d}$.

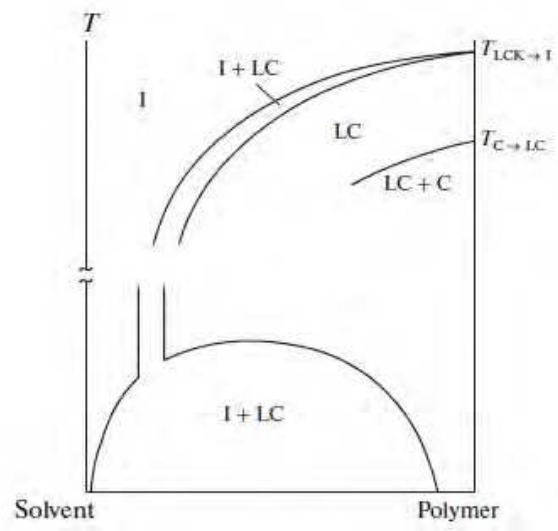

Fig. 20. Theoretical phase diagram for solutions of stiff-chain polymers according to (Flory, 1956) and (Papkov, 1992)

For rod-like macromolecules the location of the narrow biphasic region practically does not depend on temperature. But decrease of macromolecular stiffness leads to increase of the critical concentration $\varphi_{2}^{*}$ and to appearance of significant slope of the equilibrium line $\mathrm{T}^{*}\left(\varphi_{2}^{*}\right)$. Papkov, 1992 , hypothetically extended this line in direction of $100 \%$ polymer (dotted line in Fig. 20). According to his opinion, the width of the biphasic region has to be narrower at closing to $100 \%$ polymer phase, and in a limiting case crosses the temperature axis inherent for polymer. The crossing point is the temperature of thermotropic transition, i.e. 
$\mathrm{T}_{\mathrm{LC}-\mathrm{I}}$. In addition appearance of crystalline phase is possible in this region and equilibrium $(\mathrm{LC}+\mathrm{C})$ - LC should be considered.

Roughly speaking, the phase diagram for polymer systems with LC equilibrium is combination of LC-liquidus line and binodal. With increase of macromolecular flexibility the critical concentration growths and the width of biphasic domain extends. In the limiting case this phase diagram transforms to state diagram typical for amorphous equilibrium (biphasic part disappears completely).

For several polymer systems the superposition is possible of the different types of phase equilibria in various combinations: amorphous and crystalline, amorphous and LC, LC and crystalline. The principle of the mutual independence of at least two versions of phase equilibria is fulfilled (Papkov\&Kulichikhin, 1977; Papkov, 1981). In a system non-equlibrium in relation to one kind of phase equilibrium, the other kind can be realized. The sequence of phase states exchange depends on an ordering degree of forming phase. The higher ordering level, the slower a process of the new phase nuclei formation proceeds. The fastest case - formation of amorphous phases since for this case just fluctuating collision of molecules is enough without their any ordering. LC phase is more ordered that is why its nuclei form slower. Much more inhibition is observed for creation of a crystalline phase.

Do not discuss in details different partial versions of superposition of different kinds of phase equilibria there is sense to show one of the most complex to present time phase diagrams (Fig. 21). It combines LC and crystalline (CS) equilibria, as well as two binodals with UCST and LCST (Iovleva et al., 1989).

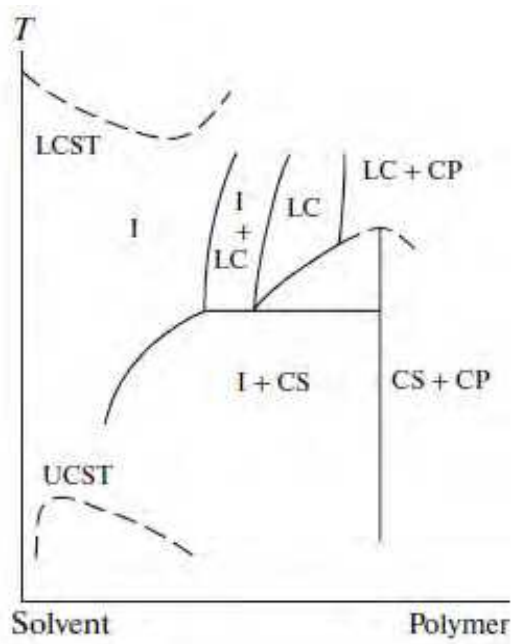

Fig. 21. Superposition of different kinds of phase equilibria for a system polymer-solvent.

In principle, an existence in one polymer a capability to form both thermotropic and lyotropic phases meets very rarely because of high values of $\mathrm{T}_{\mathrm{LC}-\mathrm{I}}$, lying higher decomposition points of polymers or difficulty to obtain very concentrated solutions for semi-stiff-chain polymers. Hydroxypropylcellulose (HPC) is one of such polymers. 


\subsection{LC and complex phase equilibria in hydroxypropylcellulose solutions}

So, HPC is among few polymers capable of forming both thermotropic and lyotropic LC phases. This is why HPC, as none other natural polymer, is accessible for analysis of phase equilibrium both in melt and solutions in a wide concentration range and in the region of easily achievable temperatures. This situation is primarily explained by the chemical structure of HPC, which contains primary and secondary hydroxypropyl and residual hydroxyl group moieties.

Therefore, a system of $\mathrm{H}$ - bonds inherent for cellulose is destabilized and the level of intermolecular interaction is reduced. As a result, the polymer shows good solubility in solvents of various nature. The above features of the chemical structure are responsible for a rather high skeletal rigidity of HPC macromolecules (the Kuhn segment length is $13 \mathrm{~nm}$ in dimethylaceteamide (Kulichikhin\&Golova, 1985), which ensures transition to the LC melt at rather low temperatures $\left(\sim 120^{\circ} \mathrm{C}\right)$ with an isotropization (clearing) temperature of $\sim 205^{\circ} \mathrm{C}$ (Makarova, 2007; Makarova et al., 2007).

HPC of the Klucel EF brand (Hercules-Aqualon, Unites States) with $\mathrm{M}_{\mathrm{w}}=8 \cdot 10^{4}$ was used. Solvents were: two oligomeric PEG samples (Spectrum, Unites States) with Mw $=400$ (PEG 400) and 1500 (PEG 1500), distilled water, propylene glycol (PG) (Labtex, Russia), triethyl citrate (TEC) (Morflex, United States), and DMSO (Gaylord Chemical Corp., United States).

\begin{tabular}{l|c|c|c|c}
\hline Solvent & Dipole moment, D & $\begin{array}{c}\text { Dielectric constant, } \\
\mathrm{F} / \mathrm{m}\end{array}$ & $\begin{array}{c}\text { Surface tension, } \\
\mathrm{mN} / \mathrm{m}\end{array}$ & $\begin{array}{c}\text { Solubility parameter } \\
\left(\mathrm{cal}^{\mathrm{c}} \mathrm{cm}^{5}\right)^{0.5}[28]\end{array}$ \\
\hline Water & 1.85 & 80 & 72.9 & 21.2 \\
DMSO & 3.96 & 47 & 44.0 & 12.8 \\
PG & 3.63 & 32 & 36.5 & 12.6 \\
TEC & 4.52 & 11 & 41.5 & 8.2 \\
PEG & 4.20 & 14 & 43.5 & 8.4 \\
\hline
\end{tabular}

Table 5. Physical-chemical characteristics of solvents used.

Table 5 lists some characteristics of the used solvents (Woolley, 1974; Kolotyrkin, 1974; Allan, 1990; Geller et al., 1996; Uusi-Penttila, 1997; Kulvinder, 2000; Gallyamov, 2009; Mali, 2007) which may be useful for analysis of phase equilibria in HPC--solvent systems. Solutions of HPC with a HPC concentration of 30, 35, 40, 45, 50, 55, 60, 70, 75, and 80 wt \% were prepared in various solvents in accordance with (Fischer et al., 1995).

The phase equilibrium in solutions was studied by the microinterference method at 18$210^{\circ} \mathrm{C}$ which renders it possible to analyze concentration profiles in the diffusion zone. On the basis of interferograms and relationships between refractive index and composition in the transition zone, the profiles of component concentration distributions were constructed and the lines of phase equilibria were derived from the boundary concentrations of components at various temperatures. To confirm the equilibrium state and reversibility of the boundary concentrations, measurements were performed at both ways: increasing and decreasing temperatures. The LC phase in solutions was identified by polarization microscopy. 
In parallel, the viscosity of solutions of different concentrations was measured. This information can be very useful for understanding not only compositions of various phases, but their properties as well.

For all systems under consideration, an increase in the concentration of HPC is accompanied by formation of the LC phase; in some cases, this process is complicated by amorphous separation of the system. In addition, for a number of systems, the CS phase can form in the high-concentration range. It is reasonable to examine various systems separately and then to generalize the data on phase equilibrium and rheological properties of HPC-based solutions.

Glycols are among the solvents used in this study. Let us first all analyze the phase equilibrium in low-molecular-weight liquids and oligomers containing terminal hydroxyl groups.

\subsubsection{HPC-PG system}

The interferograms measured at $75^{\circ} \mathrm{C}$ (Fig. 22) exhibit two phase boundaries (dotted lines): the first boundary separates isotropic and two-phase solutions, while the second boundary separates fully LC solutions from the crystalline (or the CS) phase. The presence of the LC phase is confirmed by the polarization microscopy data. Thus, under crossed polarizers the region of the LC phase of $60 \%$ solution exhibits a characteristic rainbow luminescence typical for cholesteric liquid crystals. Cellulose derivatives containing the asymmetric carbon atom (the chiral center) form just the cholesteric mesophase. As temperature is increased, the fully LC solution transforms into the two-phase region, in which LC and isotropic phases coexist. Given this, the solution gradually darkens and, after full isotropization, the field of vision becomes dark.

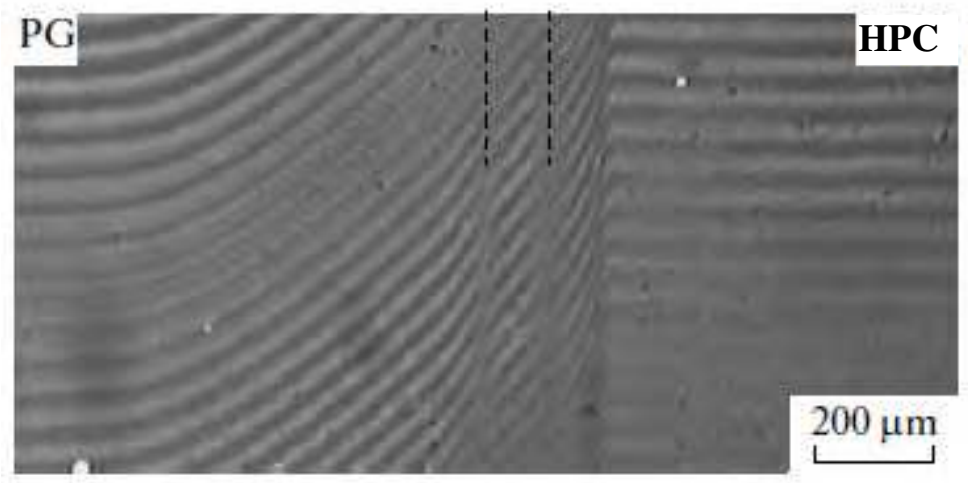

Fig. 22. Interferogram of the transition zone for the HPC-PG system at $75^{\circ} \mathrm{C}$. The phase boundaries are marked with dotted line.

The resulting phase diagram is the superposition of LC and crystalline equilibria (Fig. 23). The results of polarization microscopy measurements made it possible to refine position of the LC liquidus and to supplement the diagram with the second boundary of the two-phase corridor separating the two-phase region and the region of fully LC solutions. 


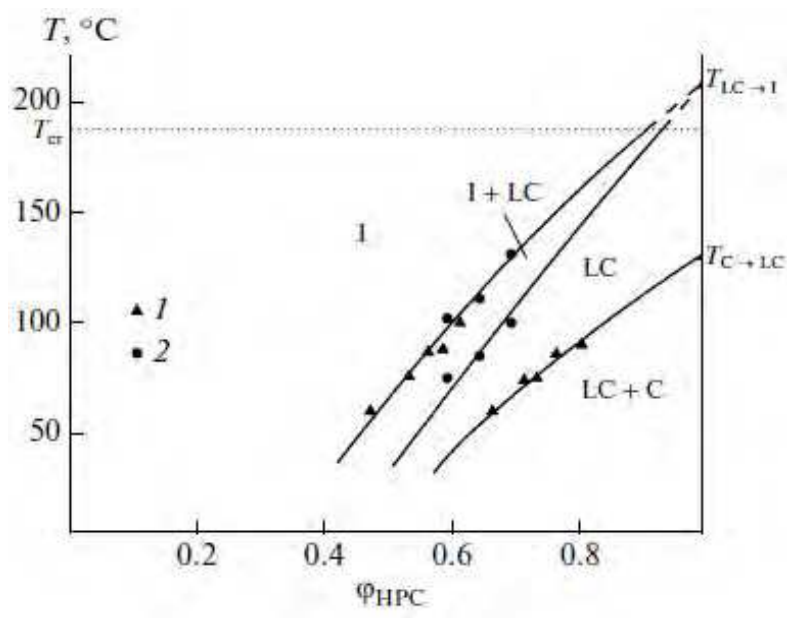

Fig. 23. Phase diagram of HPC-PG system, determined by interferometry (1) and polarizing microscopy (2). I - isotropic, LC - liquid-crystalline, C - crystalline state.

As opposed to extremely rigid-chain polymers with rod-like macromolecules, for which the I-LC transition is almost independent of temperature and concentration (the line of phase equilibrium is almost parallel to the ordinate axis), in the case of HPC solutions in PG, the position of boundary lines strongly depends on temperature and concentration of HPC. This finding is indicative of the semi-rigid nature of HPC in this solvent,

At high concentrations of the polymer, the LC phase occurs in equilibrium with the crystalline phase of HPC (C) or the CS phase. Note that in the neat HPC the degree of crystallinity does not exceed $20 \%$ (Shimamura, 1981). On the whole, the pattern of the phase diagram corresponds to the upper part of the hypothetical diagram shown in Fig. 20.

\subsubsection{HPC-PEG 400 system}

The phase diagram for HPC-PEG 400 solutions was described in (Makarova, 2007; Makarova et al., 2007). Throughout the studied temperature range, this system is characterized by LC and crystalline transitions (Fig. 24). On the left of the boundary line, the solutions are isotropic, while on the right to the boundary line, they are mesomorphic. As in the case of HPC solutions in PG, the position of the boundary line is markedly temperaturedependent.

Given almost full qualitative analogy, there are marked quantitative differences between HPC-PEG 400 and HPC-PG systems. This primarily concerns smaller critical concentration corresponding to formation of the LC phase: $25 \%$ for HPC solutions in PEG 400 compared to $\sim 40 \%$ solutions of HPC in PG. 


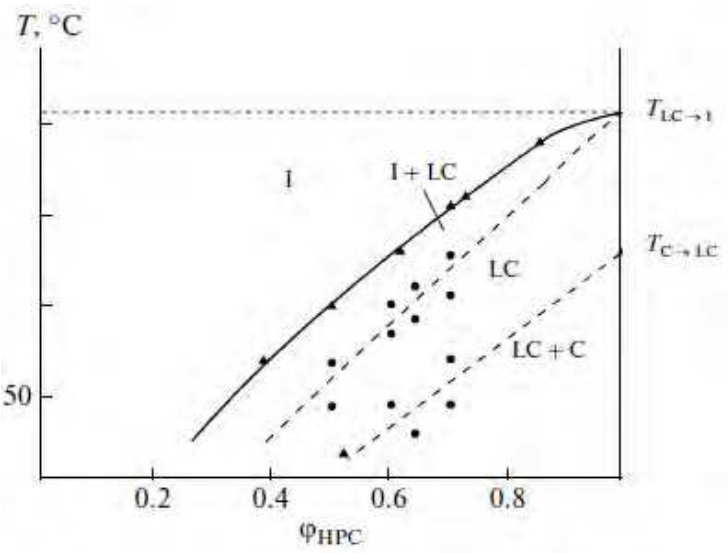

Fig. 24. Phase diagram of HPC-PEG 400 pair.

\subsubsection{HPC-PEG 1500 system}

The phase equilibrium changes substantially with an increase in the molecular weight of PEG. The main feature of HPC solutions in PEG 1500 is a superposition of LC and amorphous phase equilibria (Fig. 25) (Makarova, 2007; Kulichikhin et al., 2010; Tolstykh et al., 2010). Separation on amorphous phases is observed in the region of small and moderate concentrations with a UCMT of $\sim 185^{\circ} \mathrm{C}$, while the liquid-crystalline equilibrium occurs at higher concentrations of HPC. Within the experimental error, the position of the liquidus for a given system coincides with that for the HPC-PEG 400 system.

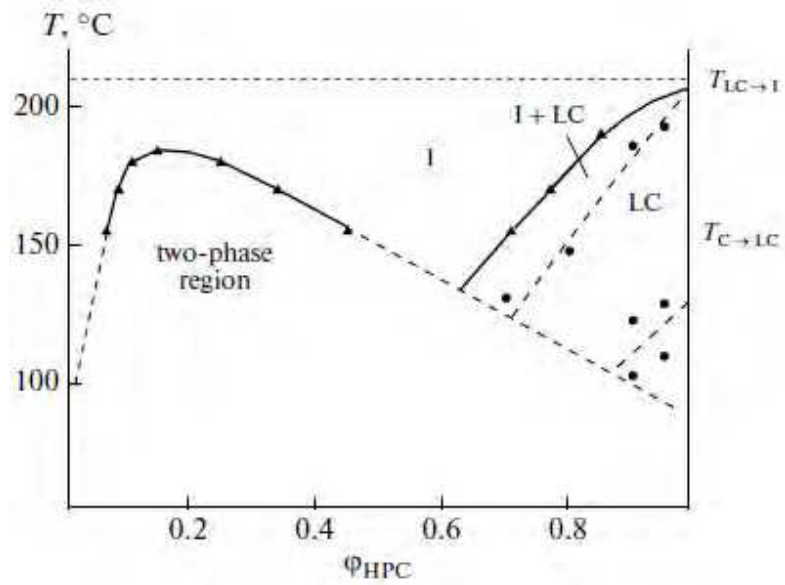

Fig. 25. Phase diagram of HPC-PEG 1500 system.

Nevertheless, PEG 1500 is a less strong solvent for HPC than PEG 400. This is evidenced by the absence of solubility in the concentration and temperature region below the binodal. Such behavior can be explained by the intramolecular association of PEG molecules, as 
confirmed by the IR data (Makarova et al., 2007). As molecular weight is increased, this effect becomes more pronounced and the probability of interaction between terminal hydroxyls of PEG and the functional groups of HPC tends to decrease.

Thus, solvents containing end hydroxyl groups dissolve well HPC and give rise to isotropic and anisotropic solutions. Owing to the presence of oxygen atoms in oligomeric PEG 400 chains and their interaction with ether or residual hydroxyl groups of HPC, the rigidity of a HPC macromolecule is somewhat higher; as a result, the transition to the LC state occurs at a lower concentration than that in the case of the low-molecular-weight PG. However, an increase in the molecular weight of PEG to 1500 causes a significant reduction in the dissolving capacity apparently due to cyclization of sufficiently long oligomeric molecules which worsens the efficiency of interaction between functional groups of the solvent and the polymer. It is not inconceivable that cyclization likewise depends on the concentration of solution since at high concentrations of HPC the LC equilibrium predominates and gives rise to LC solutions.

The hydroxyl group is also contained in a molecule of TEC, citric ester. Moreover, PEG and TEC have the highest dipole moments and the lowest dielectric permittivities among the used solvents (Table 5). Therefore, in what follows, it is advisable to examine the phase equilibrium in the HPC-TEC system.

\subsubsection{HPC-TEC system}

Fig. 26 shows the interferograms of the transition zone obtained for this system. At temperatures below $70^{\circ} \mathrm{C}$, two phase boundaries appear in the diffusion zone.
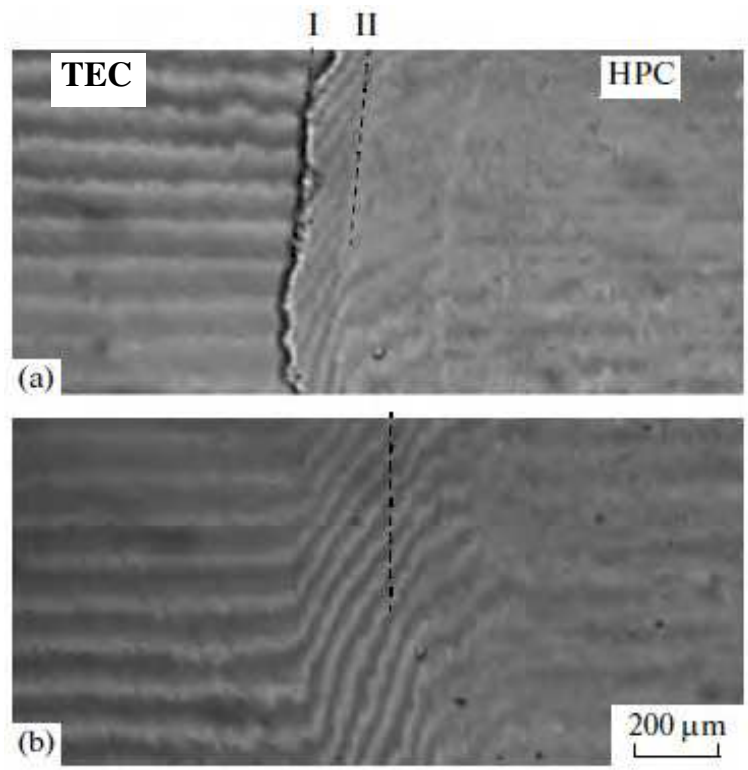

Fig. 26. Interferograms of transition zone for the HPC-TEC system registered at 55 (a) and $77^{\circ} \mathrm{C}(\mathrm{b})$. Phase boundaries are marked with dotted lines. 
One of them (I) is related to the amorphous separation of the components, while the other boundary separates isotropic and mesophase regions. Above $70^{\circ} \mathrm{C}$, phase boundary I disappears, whereas boundary II is preserved, thus indicating full dissolution of HPC in TEC at elevated temperatures with the concomitant formation of LC solutions.

On the basis of the interferograms with the use of the boundary concentrations of components estimated at various temperatures, the phase diagram was constructed (Fig. 27). As the HPC-PEG 1500 system, the HPC-TEC pair is characterized by the superposition of amorphous and LC equilibria. This situation is reflected on the diagram as the binodal with the HCST and the LC liquidus, respectively. Separation on amorphous phases is observed in the region of small and moderate concentrations, while the liquid-crystalline equilibrium is seen at high concentrations of HPC.

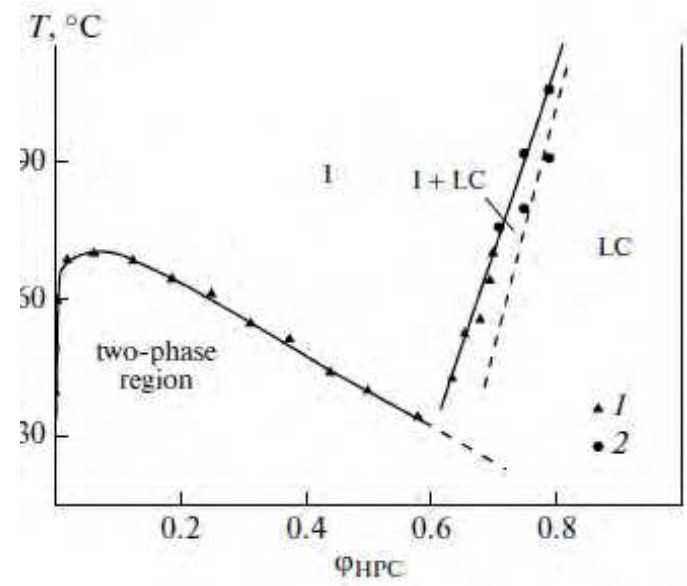

Fig. 27. Phase diagram of the HPC-TEC system constructed from data of microinterference (1) and polarizing microscopy (2)

An analysis of the phase equilibrium in the HPC-TEC system shows that this system behaves as HPC solutions in PEG 1500. It appears that TEC molecules are characterized by self-association via formation of $\mathrm{H}$-bonds between hydroxyl and ester groups. Furthermore, the steric factor may play an important role in this case. These structure features of TEC molecules affect the dissolving capacity of this solvent, which depends on solution concentration, as demonstrated by amorphous separation in the dilute-solution region and formation of the LC phase at increased concentrations of HPC.

Water is the next solvent containing hydroxyl groups, and solubility in water is a indubitable advantage of HPC. However, water possesses the lowest dipole moment and the highest dielectric constant; therefore, a certain specifics of phase equilibrium in aqueous solutions of HPC should be expected.

\subsubsection{HPC-water system}

The phase behavior of HPC-water solutions has more complex than those described above. Several versions of diagrams for this system were obtained by DSC, X-ray diffraction, NMR, 
nefelometry, and rheology. A set of some versions of diagrams is shown in Fig. 28. At temperatures below $40{ }^{\circ} \mathrm{C}$, with an increase in the concentration of $\mathrm{HPC}$, solutions transform from the isotropic region (I) to the two-phase region containing isotropic and LC phases (II) and then to fully anisotropic region (III). At high concentrations of HPC, CS (IV) forms.

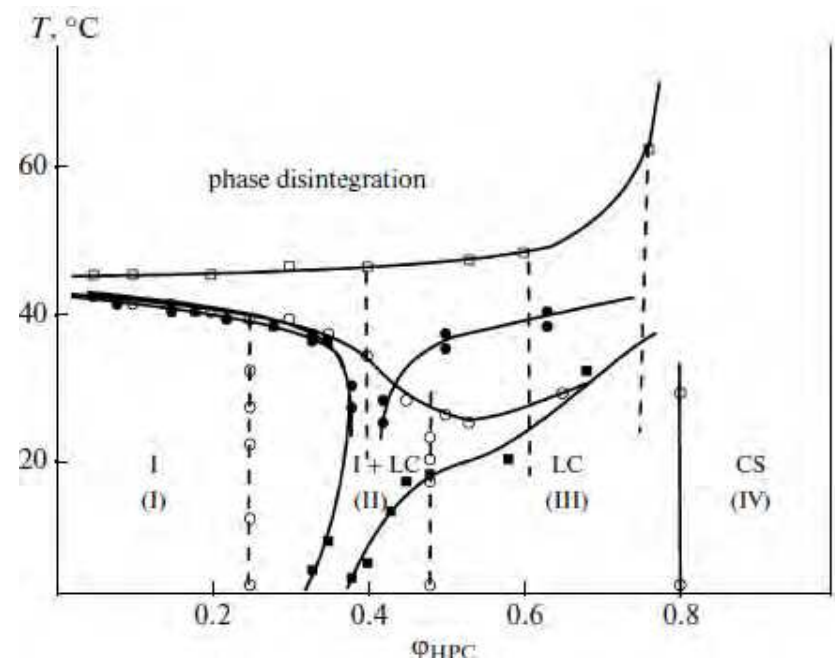

Fig. 28. A set of phase diagrams for the HPC-water system according to (Fischer et al., 1995; Guido, 1995; Larez et al., 1995; Vshivkov et al., 2007)

Although the diagrams follow the general pattern, the positions of phase equilibrium lines differ appreciably; moreover, it is unclear which is the character of phase separation at temperatures above $40-45^{\circ} \mathrm{C}$. It is known that elevated temperatures solutions experience reversible gel-formation; however, the mechanism controlling formation of the physical network remains vague up to now. Two mechanisms of this process are discussed in the literature: phase decomposition of the liquid-liquid type and formation of the HPC. $6 \mathrm{H}_{2} \mathrm{O}$ crystal solvate. Unfortunately, structural methods do not assist in understanding this mechanism. This circumstance forced us to refine the lines of phase equilibrium for this system and to gain insight into the reasons of gelation at elevated temperatures.

At temperatures below $37{ }^{\circ} \mathrm{C}$, the interferograms show all four above-mentioned regions in the transition zone (Fig. 29). As temperature is increased, the first two regions disappear and the phase boundary responsible for amorphous separation appears. The phase diagram constructed from concentration profiles in the diffusion region (Fig. 30) is the superposition of three types of phase equilibria, namely, amorphous, LC, and crystalline.

From the refined phase diagram, the mechanism of gelation of aqueous solutions at elevated temperatures becomes understandable, namely, amorphous separation and, as a result, "incomplete separation into phases" that leads to formation of the structural network. The fact that in the case of TEC and PEG 1500, solubility increases with temperature (the binodal with the HCST) and, in the case of water, decreases (the binodal with the LCST) suggest that temperature dependences of the interaction parameter are different. However, it should be mentioned that the Flory-Huggins theory cannot predict the existence of systems with the 


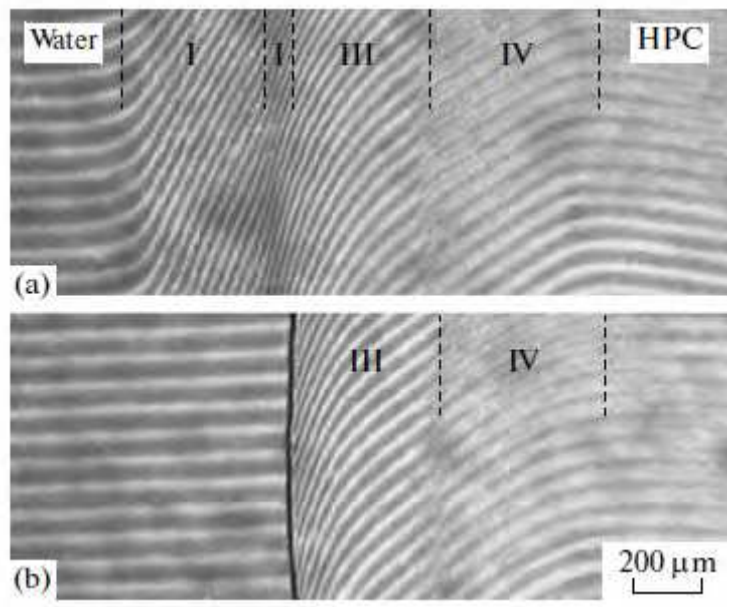

Fig. 29. Interferograms of transition zones for the HPC-water system at 29 (a) and $43^{\circ} \mathrm{C}(\mathrm{b})$. Figures denote regions corresponding to different phase states (see explanations in text).

LCST. As a rule, such phase diagrams are observed for polymer-solvent components with strong intermolecular interaction and corresponds to the upper part of the theoretical phase diagram shown in Fig. 21. In this sense, glycols and esters are more active solvents in relation to formation of $\mathrm{H}$-bonds with the functional groups of HPC than water.

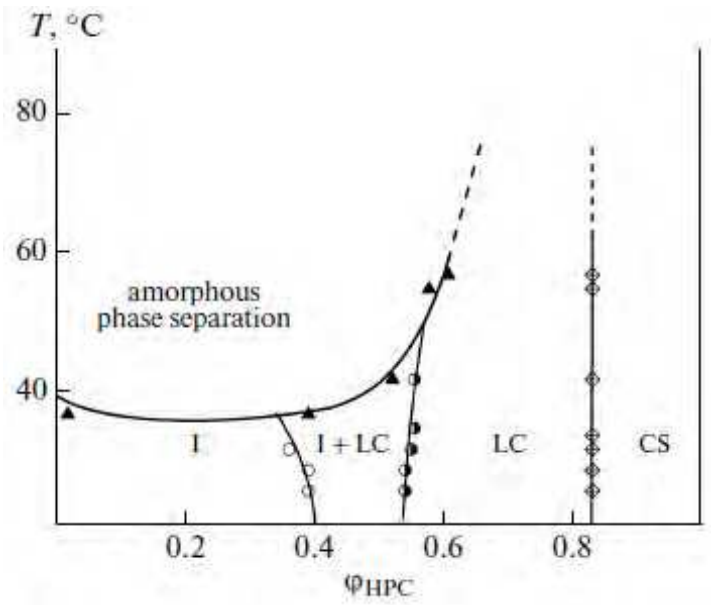

Fig. 30. The corrected phase diagram of the $\mathrm{HPC}-\mathrm{H}_{2} \mathrm{O}$ system .

The next solvent from the selected series is aprotonic dipolar DMSO, which stands somewhat apart from other solvents since it contains no hydroxyl groups. The DMSO molecule has unshared electron pairs on oxygen and sulfur atoms, and this fact explains high dissolving capacity of this donor solvent. In terms of dipole moment and dielectric constant, DMSO is rather close to PG. 


\subsubsection{HPC-DMSO system}

The typical interferogram of the region of interdiffusion of HPC and DMSO is shown in Fig. 31. Throughout the studied temperature range, the phase boundary (marked by the dotted line) is seen which separates the regions of isotropic and mesomorphic states.

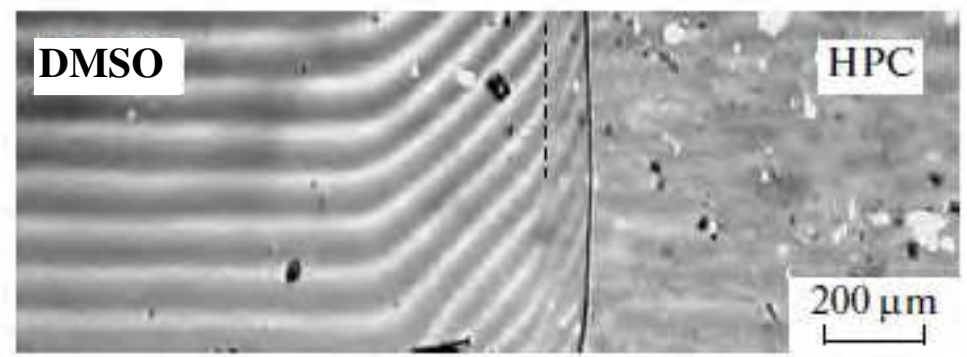

Fig. 31. Interferogram of the transition zone for the HPC-DMSO system measured at $55^{\circ} \mathrm{C}$. The phase boundary is denoted with a dotted line.

The phase diagram of the HPC-DMSO system constructed from the data of interference rheology and polarizing microscopy is characterized by the LC equilibrium (Fig. 32). Throughout the temperature range under study, the two-phase corridor manifests itself which separates the regions of isotropic and LC solutions.

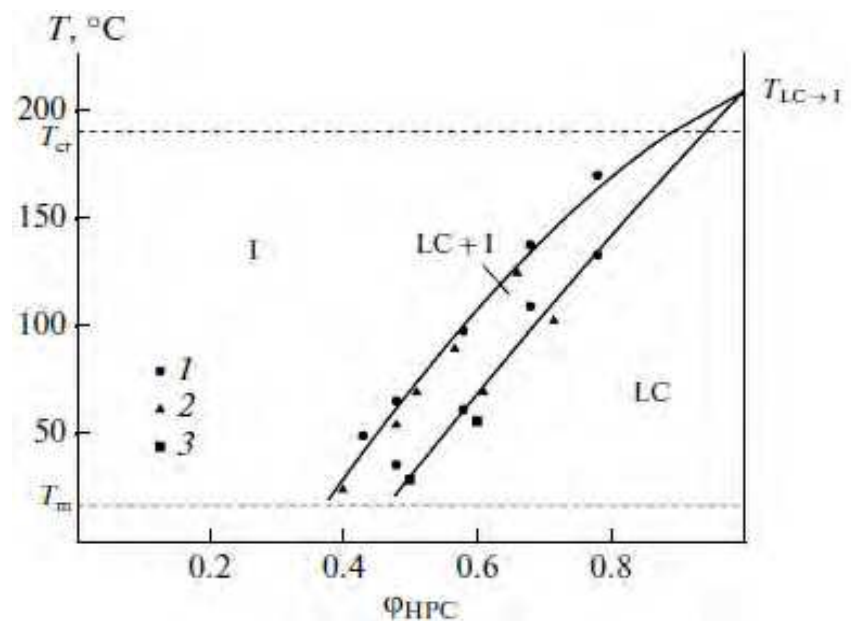

Fig. 32. Phase diagram for the HPC-DMSO system constructed from the data of microinterference (1), microscopy (2) and rheology (3).

Tables 6 and 7 list some parameters of the phase diagrams obtained at 25 and $140^{\circ} \mathrm{C}$, respectively. Let compare these data with the characteristics of the tested solvents (Table 5). Strong intermolecular interactions are responsible for the highest solubility parameter $\delta$ of water, as calculated in accordance Hildebrand and Small [62], $\delta=21.2\left(\mathrm{cal}^{\prime} \mathrm{cm}^{3}\right)^{0.5}$. This value is much higher than the $\delta$ of HPC (approximately10.72 (cal/ $\left.\mathrm{cm}^{3}\right)^{0.5}$ (Allan, 1990). 


\begin{tabular}{l|c|c|c|c|c}
\hline Solvent & $C^{*}$, vol fractions & $C^{* *}$, vol fractions & $C^{* *} / C^{*}$ & $C_{\mathrm{c}}$, vol fractions & $\begin{array}{c}\text { Critical mixing } \\
\text { temperature, }{ }^{\circ} \mathrm{C}\end{array}$ \\
\hline PG & 0.38 & 0.47 & 1.24 & 0.55 & - \\
PEG 400 & 0.25 & 0.37 & 1.48 & 0.54 & - \\
PEG 1500 & - & - & - & - & 185 (UCST) \\
PEC & - & - & - & - & 71 (UCST) \\
Water & 0.39 & 0.54 & 1.38 & 0.83 & 37 (LCST) \\
DMSO & 0.38 & 0.48 & 1.26 & - & - \\
\hline
\end{tabular}

Table 6. Critical concentrations of phase transitions for considered systems at $25^{\circ} \mathrm{C}$.

It is possible that this fact explains why HPC is not dissolved in water at temperatures above $36.5^{\circ} \mathrm{C}$ (the LCST) but it cannot explain why HPC shows excellent solubility at reduced temperatures. The latter phenomenon may be attributed to redistribution of $\mathrm{H}$-bonds formed by water molecules to those formed between water molecules and functional groups of HPC.

As regards other solvents, their solubility parameters range from 8.2 to 12.8 ; that is, they are rather close to the $\delta$ of HPC. Note that for TEC and PEG 1500, solubility parameters are somewhat smaller than the $\delta$ of HPC (8.2 and 8.4), while for PG and DMSO, they are somewhat higher (12.6 and 12.8). It is pertinent to note that for the first pair of solvents, the superposition of amorphous (with the HCST) and LC equilibria is observed, while for the second pair, only the LC equilibrium is implemented. PEG 400 stands somewhat apart from the rest solvents since in this case no amorphous separation is detected. These differences may be due to different polarizabilities of molecules: for TEC and PEG, high dipole moments coexist with low dielectric constants, while for PG and DMSO, the reverse situation is observed (disregarding water).

If we examine the critical concentrations corresponding to formation of the LC phase $\left(\mathrm{C}^{*}\right)$ and the achievement of its $100 \%$ content in the system $\left(\mathrm{C}^{* *}\right)$, then the highest rigidity of HPC macromolecules is realized in PEG, as evidenced by the lowest value of $C^{*}(0.25$ vol. fractions at $25^{\circ} \mathrm{C}$ ). In the case of water, $\mathrm{PG}$, and $\mathrm{DMSO}$, these parameters are in the range $0.38-0.39$. At a temperature of $140{ }^{\circ} \mathrm{C}$, the values of $\mathrm{C}^{*}$ are $0.64,0.70,0.73$, and 0.85 vol. fractions for PEG, DMSO, PG, and TEC, respectively.

\begin{tabular}{l|c|c|c}
\hline \multicolumn{1}{c|}{ Solvent } & $\begin{array}{c}C^{*}, \\
\text { vol fractions }\end{array}$ & $\begin{array}{c}C^{* *}, \\
\text { vol fractions }\end{array}$ & $C^{* *} / C^{*}$ \\
\hline PG & 0.73 & 0.79 & 1.08 \\
PEG 400 & 0.64 & 0.74 & 1.16 \\
PEG 1500 & 0.65 & 0.74 & 1.14 \\
TEC & 0.85 & 0.87 & 1.02 \\
DMSO & 0.70 & 0.79 & 1.13 \\
\hline
\end{tabular}

Table 7. Critical concentrations of phase transitions for considered systems at $140{ }^{\circ} \mathrm{C}$. 
For all systems, the width of the two-phase corridor, which was estimated from the ratio of critical concentrations $C^{* *} / C^{*}$, is much higher at $25^{\circ} \mathrm{C}(1.24-1.48)$ than that at $140{ }^{\circ} \mathrm{C}(1.02$ 1.16). This narrowing of the corridor with temperature, first, is indicative of the semi-rigid nature of HPC (the rigidity of chains is strongly temperature-dependent) and second, is consistent with Papkov's predictions (Fig. 20$)$ that lines $\mathrm{C}^{*}\left(\mathrm{C}_{\mathrm{HPC}}\right)$ and $\mathrm{C}^{* *}\left(\mathrm{C}_{\mathrm{HPC}}\right)$ come closer and in the limit merge on approach to the $100 \%$ polymer.

For such solvents as PG, PEG 400, PEG 1500, and water, the crystalline phase (CS or the crystalline HPC) is detected at a concentration of $\mathrm{C}_{\mathrm{c}}$. It appears that the rigidity of HPC macromolecules implemented in various solvents may affect the parameters of amorphous separation of systems. At least, this is true for HPC-PEG 1500 and HPC-TEC systems. In PEG 1500, chain rigidity, as estimated from $C^{*}$, is higher and its $\operatorname{HCST}\left(185^{\circ} \mathrm{C}\right)$ is situated at a concentration of $0.18 \mathrm{vol}$. fractions. For HPC solutions in TEC, $\mathrm{C}^{*}$ is lower and the HCST is $71^{\circ} \mathrm{C}$ at a concentration of $0.09 \mathrm{vol}$. fractions.

The above analysis does not pretend to be full and does not reveal all features of phase equilibrium in HPC-solvent systems. Nevertheless, it demonstrates the peculiar phase behavior of these systems in relation to the structure and properties of solvents.

\subsubsection{Rheological properties of HPC solutions}

The rheological properties of LC systems are characterized by some distinctive features (Papkov et al., 1974; Kulichikhin et al., 1982; Kulichikhin, 1989). As opposed to isotropic polymers, LC polymers manifest the abnormal viscosity throughout the studied shear rate range. This effect is associated with the polydomain structure of LC systems and the presence of a system of disclinations at rest. With an increase in the shear rate as a result of orientation processes, the system gradually transforms into the monodomain structure. Furthermore, in the two-phase region, interphase boundaries between drops of isotropic phase and LC disperse medium, or LC drops and isotropic matrix make a substantial contribution to the rheological response.

The main result at analysis of rheological properties of solutions under consideration consists in conclusion that viscosity is a parameter sensitive to LC transitions. The flow curves reflect the existence of the polydomain structure in the initial LC system and its transformation into the monodomain structure with an increase in the intensity of shear deformation. Presence of polydomain structure and such defects as disclinations is stipulated the viscoplastic behavior with a yield stress. However, the most vivid dependence that makes it possible to estimate the critical concentrations of transitions is the concentration dependence of viscosity. In this sense rheological data are good addition to results of interferometric measurements to prove the location of boundary lines between liquid phases.

Fig. 33 displays dependences of this type for several systems under study in the region of the Newtonian (isotropic solutions) or quasi-Newtonian (anisotropic solutions) behavior. As is seen, these dependences contain extreme points. Despite of different scales of maxima and minima, extremes are observed for all systems. As is seen from comparison with phase diagrams, the maximum of viscosity corresponds to the critical concentration, at which the anisotropic phase appears in solutions. When the system contains isotropic and LC phases, an increase in the fraction of the anisotropic phase causes a drop in viscosity. The minimum 

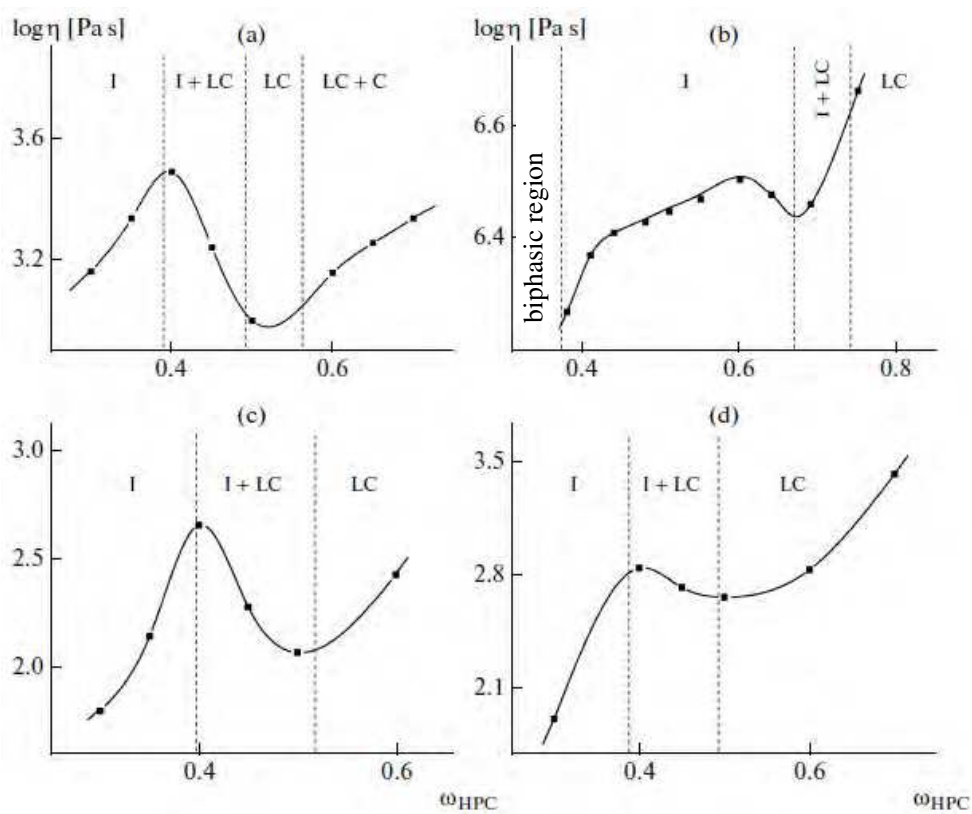

Fig. 33. Concentration dependences of viscosity for solutions of HPC in PG at 23 (a), TEC at 50 (b), water at 23 (c), DMSO at $23^{\circ} \mathrm{C}(\mathrm{d})$. Dotted lines show phase boundaries according to phase diagrams constructed.

on the curve corresponds to the critical concentration at which the system becomes fully LC. After further increase in concentration, viscosity increases again due to a reduction in the free volume. Thus, an increase in viscosity with concentration is typical for single-phase (isotropic and LC) solutions, whereas in the two-phase corridor, viscosity drops with increase of concentration (the fraction of the LC phase).

It is pertinent to note the specifics inherent in the HPC-TEC system in the concentration range neighboring amorphous phase separation. In this case, the abnormal decrease in viscosity with a decrease in the concentration of HPC is apparently related to formation of nuclei (species) of the amorphous phase highly concentrated with respect to the polymer.

The above dependences of viscosity on concentration vividly reflect the specifics inherent in solutions of rigid-chain polymers, which form the LC phase in a certain temperatureconcentration range. Exactly for these systems viscosity is as a parameter suitable to refine the position of boundary lines of phase equilibrium. Very likely this is the key point in a given analysis. The region of amorphous equilibrium is practically unfeasible for the correct study by means of rheological methods since the system experiences phase separation below the binodal.

\section{Conclusion}

The different kinds of phase equilibria (amorphous, crystalline, liquid-crystalline) are considered. Each of them has definite futures that were analyzed on examples of various 
polymer-polymer and polymer-solvent systems. All above mentioned results relate to thermodynamic properties for binary systems containing polymers, and all of them were obtained by interferometry method. In addition to thermodynamic data, this method gives information about kinetics of components interactions that allows us to measure simultaneously interdiffusion coefficients. This dualism of optical interferometry method in application to interaction in polymer containing systems is its great advantage. That why its application in scientific practice is very useful and fruitful.

The couple PC - low molecular weight PMMA is a good example of polymer system with amorphous phase equilibrium. Method of interferometry is very useful and fruitful for such kind of systems due to informativity and combination of joint approaches: kinetics of masstransfer and thermodynamics of phase states. Increase of the mutual solubility with temperature indicates on UCST that could not be measured experimentally but was calculated using evolution of the interaction parameter with concentration.

Comparison of the phase diagram with concentration dependence of viscosity allowed us to observe local minimum of viscosity near the binodal (sooner in meta-stable region between binodal and spinodal where microemulsion appears as the first step of the phase decomposition) and to apply the empiric equation for calculation of the blends viscosity by means of accurate determination of the composition from binodal branches. Joint consideration of diffusion and rheological properties seems to be important for judging about structure, kinetic and thermodynamic behaviors of complex, multiphase polymer systems. This approach will be used further for analysis of systems with LC and crystalline equilibria.

Calculations of the interphase layers thickness depending on dimensions of drops and time, based on knowledge of interdiffusion coefficients can be very useful for correct organization of technological process of mixing and, consequently, development of new materials with three-phase structure and new properties.

Several examples of crystalline equilibrium in couples polyester-polyester are considered a well. The neat polymers are semi-crystalline and phase equilibrium for their pairs is describing by liquidus lines, sometimes with eutectic points. But this situation takes place at $\mathrm{T}<280-290{ }^{\circ} \mathrm{C}$, when chemical interaction can be neglected. The original feature of these data consists in using as one component of pair thermotropic LC polyesters and copolyesters. Up to clearing point of LC component the interdiffusion process is registered using one part of the interference patterns. Such approach was demonstrated first time.

At using LC polymer melt as one component, both interdiffusion process and ester-ester exchange is delayed because of high molecular orientation in LC polymer along interface. This dense layer prevents fast penetration of coil-like macromolecules of isotropic polyester melt. But in the case of PET-PEN pair both melts are isotropic and transparent, that is why interaction process can be visualized easy. The hypothetic mechanism of joint action of physical and chemical interaction is proposed. The first stage is diffusion, but after penetration of macromolecules of one polymer into another the trans-esterification starts and transition between these two processes can be estimated from kinetics of movement of iso-concentration planes.

Method of optical interferometry is not so often used for describing phase equilibria in solutions of stiff-chain polymers forming in solutions LC phase. In this part was shown its application for several solutions the same polymer in different solvents. We were wonder to 
informativity of interferometry for such systems. The main portion of equilibria lines in solutions were detected by interferometric method. Of course, for LC systems method of polarizing microscopy has to be obligatory supporting method for confirmation of the LC phase existence. In addition, viscometry seemed to be very useful again not only for description of features of these systems at flow, but also for additional detection of LC transitions as well as for formation of CS phase.

As opposed to diagrams obtained for other systems, for which two types of phase equilibrium are located in different concentration regions, in the case of aqueous systems, all transitions, including formation of crystal solvates, take place below the convex part of the binodal, that is, at the same concentrations but at different temperatures. This result indicates that the activity of water with respect to formation of hetero $\mathrm{H}$-bonds tends to increase with a decrease in temperature.

\section{Acknowledgement}

Authors appreciate the partial financial support from Russian Foundation for Basic Research (grants 08-03-12035, 09-03-00285a, 10-03-00994a, 11-03-12042-ofi-m).

\section{References}

Allan, F.M., (1990). Barton Handbook of Polymer-Liquid Interaction Parameters and Solubility Parameters. CRC Press LLC, ISBN: 0095-9782, Florida

Avdeev, N.N. (1990). Phase equilibria and diffusion in oligomer-polymer systems. PhD Thesis. Moscow, Institute of Physical Chemistry

Avdeev, N.N., Makarova, V.V., Kotomin, S.B., Kulichikhin, V.G. \& Kuleznev, V.N., (2001). Compatibility and viscosity of polycarbonate- poly(methyl methacryalate) blends, Polymer Science, Ser. A, Vol. 43, №2, 112-117, ISSN: 1560-0904

Borovskii, I.; Gurov, K.; Marchukova, I. \& Ugaste, Yu. (1973). Processes of interdiffusion in alloys, Nauka, Moscow (in Russian) ISBN:

Butzbach, G. D. \& Wendorff, J. (1991). Polycarbonate-poly(methyl methacrylate) blends: the role of molecular interactions on miscibility and antiplasticization, Polymer, Vol. 32, № 7, 1155-1159, ISSN: 0032-3861

Chalykh, A.E. \& Gerasimov, V.K. (2004). Phase equilibria and phase structure of polymer blends. Uspekhi khimii, Vol.73, №1, 63- (in Russian), ISSN: 0042-1308

Chalykh, A.E., Gerasimov, V.K. \& Mikhailov, Yu.M. (1998). Diagrams of Phase State of Polymer Systems, Yanus-K, Moscow (in Russian), ISBN: 5-8037-0016-9

Chalykh, A.E., Aliyev, A.D. \& Rubtsov, (1990). Electronic Probe Microanalysis in Polymer Investigation, Nauka, Moscow (in Russian)

Chiou, J. S., Barlow, J. W., \& Paul, D. R. (1987). Miscibility of bisphenol-A polycarbonate with poly(methyl ethacrylate). J. Polym. Sci., Polym. Phys. Ed., Vol. 25, № 7, 1459-1471, ISSN 0887-6266

Collyer, A, (1996). Introduction to Liquid Crystal Polymers. In Rheology and Processing of Liquid Crystal Polymers, Acierno, D., Collyer A.A. (Eds.). Chapman\&Hall, ISBN: 0412596407

Crank, J. (1956). The Mathematics of Diffusion. Clarendon Press, ISBN: 0198534116, Oxford 
Debier, D., Devaus, J. \& Legras, R. (1994). Blends of bisphenol a polycarbonate and acrylic polymers. I. A chemical reaction mechanism. J. Polym. Sci., Polym. Phys. Ed., Vol. 33, № 3, 407-414, ISSN: 0887-624X

Eastmond, G.C. \& Kotomin, S.V. (1994). Spectroscopic monitoring of phase separation in polymer blends. Polymer, Vol. 35, № 4, 882-884, ISSN: 0032-3861

Fischer, H., Murray, M., Keller, A. \& Odell J.A. (1995). On the Phase Diagram of the System Hydroxypropylcellulose-Water. J. Mater. Sci. Vol. 30, № 10, 4623-4627, ISSN: 00222461

Flory, P.J. (1956). Phase diagrams of rod-like polymer solutions, Proc. Roy. Soc., Ser. A., Vol. 234, № 1, 73-81

Gallyamov, M.O., (2009). PhD Thesis, Moscow State University

Geller, B.E., Geller, A.A. \& Chirtulov, V.G. (1996). Practicum on Physical Chemistry of Fiberforming Polymers. Khimiya, ISBN 5-7245-0944-X, Moscow

Guido, S. (1995). Macromolecules, Vol. 28, № 13, 4530-4539, ISSN: 0024-9297

Guo, M. \& Brittain, W. J. (1998). Structure and Properties of Naphthalene-Containing Polyesters. 4. New Insight into the Relationship of Transesterification and Miscibility. Macromolecules, Vol. 31, 7166-7172, ISSN: 0024-9297

Ihm, D. W.S., Park, Y., Chang, C. G., et al., (1996). J. Polym.Sci., Part A: Polym. Chem., Vol. 34, 2841- 2849, ISSN: 0887-6266

Iovleva, M.M. \& Papkov, S.P. (1982). Polymer Crystal Solvates. Polymer Science, Ser. A, Vol.24, № 2, 233-237, ISSN:1560-0904

Iovleva, M.M., Smirnova, V.N. \& Prozorova, G.E. (1989). Upper and Lower Critical Solution Temperatures for Solutions of Stiff-Chain Aromatic Polyamides. Polymer Science, Ser. B., Vol. 31, №11, 861- 865, ISSN: 1560-0904

Kim, C. K. \& Paul, D. R. (1992). Effects of polycarbonate molecular structure on the miscibility with other polymers. Macromolecules., Vol. 25, № 12, P. 3097- 3105, ISSN 0024-9297

Klenin, V.I. (1995). Thermodynamics of systems with flexible-chain polymers, Saratov University Publ., ISBN: 5-292-01936-4, Saratov (in Russian)

Koningsveld, R. \& Kleintjens, L.A. (1977). Thermodynamics of polymer mixtures. J. Polym. Sci., Polym. Symp. №61, 221-249. ISSN: 0887-6258

Kolotyrkin, Ya.M. (1974). Electrochemistry of Metals in non-Aqueous Solutions, Mir, Moscow

Kotomin, S.V. \& Kulichikhin,V.G. (1996). Application of Squeezing Flow Technique for Viscosity Measurements of Polymer Liquids. Polymer Science, Ser.B, Vol.38, №12, 2079- ISSN: 1560-0904

Kuleznev, V.N. (1980). Polymer Blends, Khimiya, Moscow (in Russian), ISBN: 5-7245-0944-X

Kuleznev, V.N. \& Kandyrin L.B. (2000). Structure-Rheology Behavior of Binary Polymer Blends in Vicinity of Phase Decomposition, Polymer Science, Ser.B. Vol. 42, №4, 711714. ISSN:1560-0904

Kuleznev, V.N. (1987). Features of Structure and Properties of Polymer Blends near Decomposition Region, Colloid. J., Vol. 49, №5, 881-890, ISSN 0023-2912

Kulichikhin, V.G. \& Golova, L.K., (1985). Liquid Crystal State in Solutions of Cellulose Derivatives. Khimiya Drevesiny (Chemistry of Wood), № 3, 9-17, ISSN: 0201-7474

Kulichikhin, V.G. (1989). Rheology, Phase Equilibria and Processing of Lyotropic Liquid Crystalline Polymers. Mol. Cryst. Liq. Cryst., Vol. 169, 51-81, ISSN 0026-8941

Kulichikhin, V.G., Avdeev, N.N., Semakov, A.V., Plate, N.A. (1994). Interphase Interaction in Heterogeneous Polymer Systems Containing Liquid-Crystal Component. Russian Chemical Bulletin, Vol. 43, №11, 1753-1769, ISSN 0002-3353 
Kulichikhin V.G., Makarova V.V., Tolstykh M.Yu., Basil'ev G.B., (2010), Phase Equilibria in Solutions of Cellulose Derivatives and Rheological Properties of Solutions in Various Phase States. Polymer Science, A, V.52, №11, P.2001-2013. ISSN: 1560-0904

Kulichikhin, V.G., Kudryavtsev, G.I. \& Papkov S.P. (1982). Rheological Properties of LiquidCrystalline Polymer Solutions, Int. Polym. Mater, Vol. 9, № 3-4, 239-256, ISSN: 00914037

Kulvinder, K., (2000). PhD Thesis, Jai Narain Vyas University, Jodhpur, India

Kyu, T., Ko, C.-C., Lim, D.-S., Smith, S. D. \& Noda, I. (1993). Miscibility studies on blends of polycarbonate with syndiotactic polymethyl methacrylate. J. Polym. Sci., Polym. Phys. Ed., Vol. 31, № 11, 1641-1648, ISSN: 0887-6266

Landry, Ch. J. T. \& Henrichs, P. M. (1989). The influence of blending on the local motions of polymers. Studies involving polycarbonate, poly(methyl methacrylate), and a polyester. Macromolecules, Vol. 22, № 5, 2157-2166, ISSN: 0024-9297

Larez, V., Crescenzi, V. \& Ciferri A. (1995). Phase separation of Rigid Polymers in Poor Solvents. 1. (Hydroxypropyl)cellulose in Water. Macromolecules, Vol. 28, № 15, 5280-5284, ISSN: 0024-9297

Lipatov, Yu.S. (1984). Phase Diagrams of Polymer Solutions and Blends, Naukova Dumka, Kiev (in Russian)

Litmanovich, A. D., Plate, N. A. \& Y. V. Kudryavtsev, (2002). Reactions in Polymer Blends: Interchain Effects and Theoretical Problems. Prog. Polym. Sci. Vol. 27, № , 915- ISSN: 0079-6700

Makarova, V.V. (2007). Mass-transfer in vicinity of interfaces in polymer-polymer systems. PhD Thesis. Moscow, A.V.Topchiev Institute of Petrochemical Synthesis

Makarova, V. V., Avdeev, N. N., Strelets, B. Kh., et al., (2005). Approaches to Chemical and Physical Modification of Polyethylenterephthalate. Polymer Science, Ser. A, Vol. 47, №7, 1140-1152 , ISSN:1560-0904

Makarova, V.V., Gerasimov, V.K., Tereshin, A.K., Chalykh, A.E. \& Kulichikhin, V.G. (2007). Diffusion and Phase Behavior of Hydroxypropylcellulose-Polyethylenglycole System. Polymer Science, Ser. A, Vol. 49, №4, 663-673, ISSN: 1560-0904

Mali, C.S., Chavan, S.D., Kanse, K.S., Kumbharkhane, A.C. \& Mehrotra S.C. (2007). Indian J. Pure Appl. Phys., Vol. 45, № 5, 476-480, ISSN: 0019-5596

Malkin, A.Ya, Chalykh, A.E., Kovriga, V.V., Askadsky, A.A. (1983). Experimental Methods of Polymer Rhysics: Measurement of Mechanical Properties, Viscosity and Diffusion, Prentice Hall, New Jersey, ISBN-10: 0132954850

Malkin, A.; \& Chalykh, A. (1979). Diffusion and viscosity of polymers. Methods of measurement, Khimiya, Moscow (in Russian) ISBN: 5-7245-0944-X

Nishimoto, M., Keskkula, H. \& Paul, D. R. (1991). Role of slow phase separation in assessing the equilibrium phase behaviour of PC-PMMA blends. Polymer, Vol. 32, № 2, 272278, ISSN: 0032-3861

Okamoto, M. \& Kotaka, T., (1997). Phase separation and homogenization in poly(ethylene naphthalene-2,6-dicarboxylate)/ poly(ethylene terephthalate) blends. Polymer, Vol. 38, № ,1357-1363, ISSN: 0032-3861

Palatnik, L.C. \& Landau, A.I., (1961) Phase Equilibria in Multicomponent Systems, Kharkov University Publ., Kharkov (in Russian)

Papkov, S.P. (1981). Phase equilibrium in polymer-solvent system, Khimiya, Moscow (in Russian), ISBN: 5-7245-0944-X

Papkov, S.P. (1971) Physical-chemical Fundamentals of Polymer Solutions Processing, Khimiya, Moscow (in Russian), ISBN: 5-7245-0944-X 
Papkov, S.P., Kulichikhin, V.G., Kalmykova, V.D. \& Malkin A.Ya. (1974). Rheological properties of anisotropic poly(para-benzamide) solutions, J. Polym. Sci., Polym. Phys. Ed. Vol. 12. № 9. 1753-1759, ISSN: 0887-6266

Papkov, S.P. Phase Equlibria in Polymer Systems Containing a Liquid-Crystalline Phase. (1992). In: Liquid-Crystal Polymers. Plate, N.A. (Ed.) 39-70. Plenum Press, ISBN: 0306-44219-1, New York

Papkov, S.P., Iovleva, M.M., Smirnova, V.N. \& Volokhina, A. V. (1986). Experimental Analysis of the Phase equilibrium in the System Stiff-Chain Polyamide - Sulfuric Acid. Polymer Science, Ser. B, Vol. 28, № 9, 677-681,ISSN:1560-0904

Papkov, S.P., Kulichikhin, V.G. (1977). Liquid Crystalline State in Polymers, Khimiya, Moscow (in Russian), ISBN: 5-7245-0944-X

Rabeony, M., Hseih, D. T., Garner, R. T. \& Peiffer, D. G. (1992). Nearly monodisperse spherical domain morphology in polycarbonate/poly (methylmethacrylate) blends following spinodal decomposition coupled with an interchange reaction. J. Chem. Phys., Vol. 97, № 6, 4505-4512, ISSN: 0021-9606

Scott, R. L. (1949). The Thermodynamics of High Polymer Solutions. V. Phase Equilibria in the Ternary System: Polymer 1-Polymer 2-Solvent. J. Chem. Phys. ,Vol. 17, № 2, 279-285, ISSN: 0021-9606

Shimamura, K., White, J.L. \& Fellers J.F. (1981). Hydroxypropylcellulose, a Thermotropic Liquid Crystal: Characteristics and Structure Development in Continuous Extrusion and Melt Spinning. J. Appl.Polym. Sci. Vol. 26, № 7, 2165-2180. ISSN: 0021-8995

Stewart, M. S., Cox, A. J. \& Naylor, D. M. (1993). Reactive Processing of Poly(ethylene 2,6naphthalene dicarboxylate)/Poly(ethylene terephthalate) blends. Polymer, Vol.34, 4060-4067, ISSN: 0032-3861

Tager, A.A. (1978). Physical Chemistry of Polymers, Khimiya, Moscow (in Russian), ISBN: 57245-0944-X

Tereshin, A.K., Vasil'eva, O.V., Avdeev, N.N., Bondarenko, G.N. \& Kulichikhin, V.G. (2000). Effects of Interphase Interactions on Rheological Properties of PET-LC Polyester Blends. Polymer Science, Ser. A, Vol.42, №6, 1009-1015, ISSN:1560-0904

Tolstykh, M., Makarova, V., Semakov, A. \& Kulichikhin, V. (2010). Polymer Science, Ser. A, Vol. 52, № 2, 228-234, ISSN: 1560-0904

Tompa, H. (1956). Polymer Solutions. Academic Press, ASIN: B0006AUTUA, New York

Transreactions in Condensation Polymers, (1999). Ed. By Fakirov, S.,Wiley, ISBN: 9783527297900, Weinheim

Uusi-Penttila, M.S., Richards, R.J., Torgerson, B.A. \& Berglund, K.A. (1997). Ind. Eng. Chem. Res., Vol. 36, № 2, 510-512, ISSN: 0888-5885

Vshivkov, C.A., Adamova, L.V., Rusinova, E.V., Safronov A.P., Dreval, V.E. \& Galyas A.G., (2007). Thermodynamics of Liquid-Crystalline Solutions of Hydroxypropyl-cellulose in Water and Ethanol. Polymer Science, Ser. A, Vol. 49, № 5, 578-583, ISSN: 1560-0904

Woo, E. M. \& Su, C. C. (1996). Phase micro-heterogeneity in bisphenol-A polycarbonate/ poly(methyl methacrylate) blends. Polymer, Vol. 37, № 18, 4111-4118, ISSN: 00323861

Woolley, E.M. \& George, R.E. (1974). Ionization constants for water and for very weak organic acids in aqueous organic mixtures. J. Solut. Chem., Vol. 3. № 2. 119-126, ISSN: 00959782 


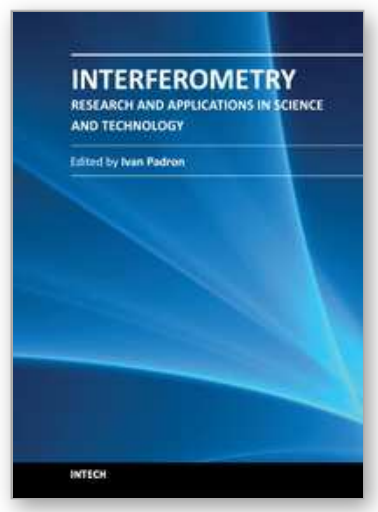

\section{Interferometry - Research and Applications in Science and Technology}

Edited by Dr Ivan Padron

ISBN 978-953-51-0403-2

Hard cover, 462 pages

Publisher InTech

Published online 21, March, 2012

Published in print edition March, 2012

This book provides the most recent studies on interferometry and its applications in science and technology. It is an outline of theoretical and experimental aspects of interferometry and their applications. The book is divided in two sections. The first one is an overview of different interferometry techniques and their general applications, while the second section is devoted to more specific interferometry applications comprising from interferometry for magnetic fusion plasmas to interferometry in wireless networks. The book is an excellent reference of current interferometry applications in science and technology. It offers the opportunity to increase our knowledge about interferometry and encourage researchers in development of new applications.

\section{How to reference}

In order to correctly reference this scholarly work, feel free to copy and paste the following:

Veronica Makarova and Valery Kulichikhin (2012). Application of Interferometry to Analysis of Polymer-Polymer and Polymer-Solvent Interactions, Interferometry - Research and Applications in Science and Technology, Dr Ivan Padron (Ed.), ISBN: 978-953-51-0403-2, InTech, Available from:

http://www.intechopen.com/books/interferometry-research-and-applications-in-science-andtechnology/application-of-interferometry-to-analysis-of-polymer-polymer-and-polymer-solvent-interactions

\section{INTECH}

open science | open minds

\section{InTech Europe}

University Campus STeP Ri

Slavka Krautzeka 83/A

51000 Rijeka, Croatia

Phone: +385 (51) 770447

Fax: +385 (51) 686166

www.intechopen.com

\section{InTech China}

Unit 405, Office Block, Hotel Equatorial Shanghai

No.65, Yan An Road (West), Shanghai, 200040, China

中国上海市延安西路65号上海国际贵都大饭店办公楼 405 单元

Phone: +86-21-62489820

Fax: +86-21-62489821 
(C) 2012 The Author(s). Licensee IntechOpen. This is an open access article distributed under the terms of the Creative Commons Attribution 3.0 License, which permits unrestricted use, distribution, and reproduction in any medium, provided the original work is properly cited. 